Portland State University

PDXScholar

\title{
Electron optical study of a secondary electron multiplier
}

Chang Min Shen

Portland State University

Follow this and additional works at: https://pdxscholar.library.pdx.edu/open_access_etds

Part of the Atomic, Molecular and Optical Physics Commons, Optics Commons, and the Plasma and Beam Physics Commons

Let us know how access to this document benefits you.

\section{Recommended Citation}

Shen, Chang Min, "Electron optical study of a secondary electron multiplier" (1970). Dissertations and Theses. Paper 1478.

https://doi.org/10.15760/etd.1477

This Thesis is brought to you for free and open access. It has been accepted for inclusion in Dissertations and Theses by an authorized administrator of PDXScholar. Please contact us if we can make this document more accessible: pdxscholar@pdx.edu. 


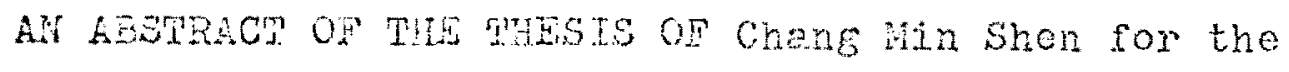
Haster of Science in Physics presented July 24, 1970.

Title: Blectron Optical Btudy of a Secondary Electron Multipien.

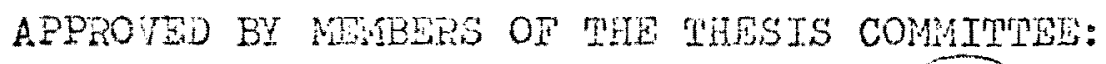

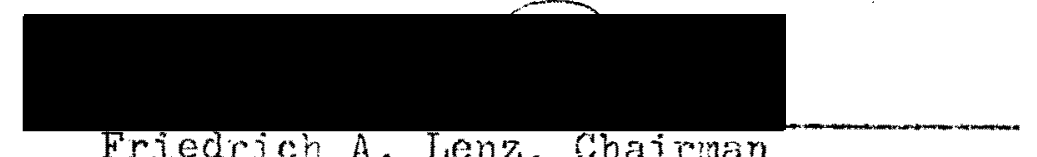

Friedrich A. Lenz, Chajrman

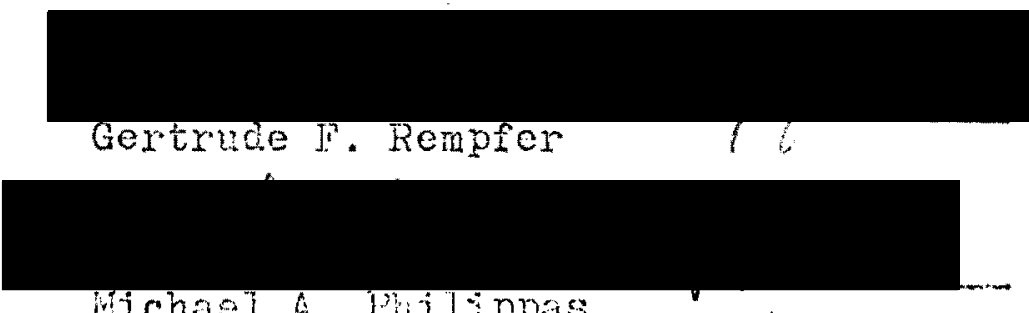

Wichae A A Pilippes

Electron optical theory was applica to the design of the geometrical structure of an electron multipliex for an jmage intensifier. A special structure satisfying troduction requirenents vas studied. Electron opticel calculations consisted of deteraining the potential distribution and tracing the electron trajectories. Iilebman's procedure was used to solve lavlaco's equation with constart potentials on the multiplier electrodes as boundary conditions. The trajectories were deternined by solving the equation of notion in an 
electrostatic field using a Runge-Kutta procedure. The initjal conditions for the trajectories were the Initial energies, initial positions, and the initial directions of the secondary electrons. The plotted trajectories indicated the feasibility of an electron nultiplier of the type studied. 


\title{
ELECTRON OPTICAL STUDY \\ OF A \\ SECONDARY ELECTRON MULP IPLIER
}

by

CHANG MIN SHEN

\begin{abstract}
A thesis submitted in partial fulfillment of the requirements for the degree of

MASTER OF SCIENCE

in

PHYSICS
\end{abstract}

Portland State University

1970 
TO THE ORTCE OF GRADUAE SWUDSE:

The menbers of the comitie anprove the thesis of Changin Shen presented July $24,1970$.

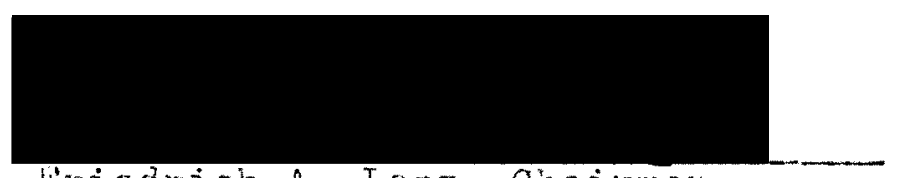

Friedrich A. Lenz, Chairman

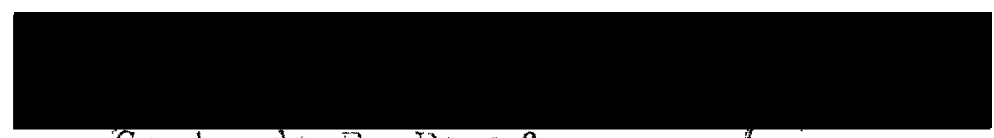

Gertrude F. Remofer

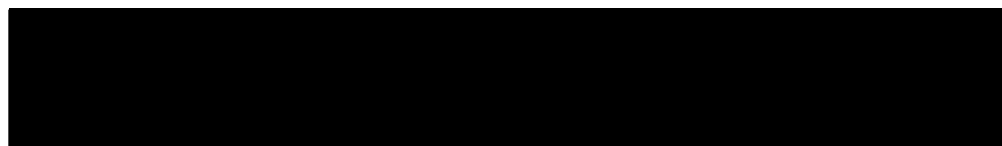

Michael A. Pilinas

APPOOVEO

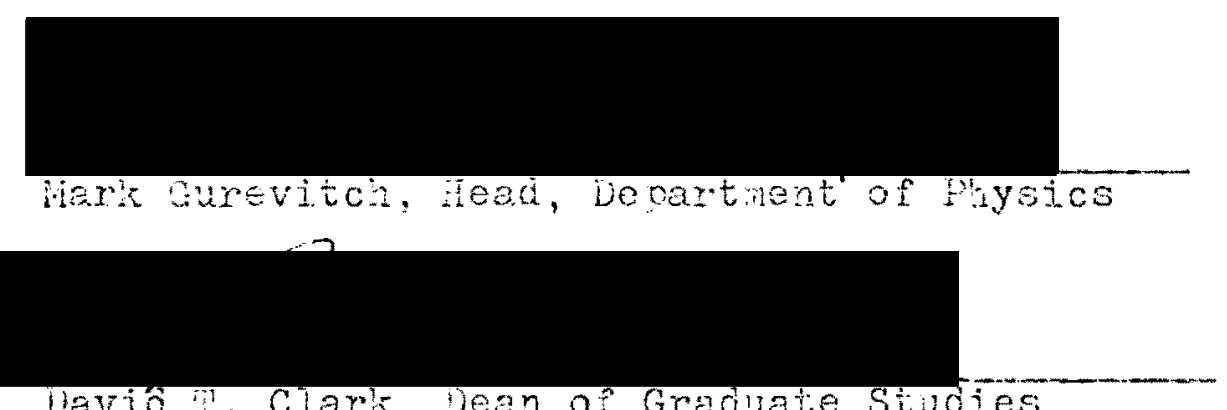

Davia in. Clark, Dean of Graduate Studies 
TABIEE OF CONTEITS

PAGE

LIST OF FIGURES . . . . . . . . . IV

INTRODUCTION . . . . . . . . . . . 1

THE SECONDARY EMISSION PROPERTIES

OF THE KULTIPLIER . . . . . . . 11

THE DETERIINATION OF THE FOTENTIAL

DISTRIBUTION

THE PLOT OF THE TRAJECTORIES . . . . . . . 25

CONCLUSION . . . . . . . . . . . . . 40

EIBLIOGRAPHY . . . . . . . . . 4 41

APFEINDICES ............. 42 


\section{LIST OF FIGURES}

FIGURE

PAGE

1 Structure of the Multiplier. . .

2. Shape and Dimensions of the Multiplier. . . . . . . . 6

3 Local Gain of the Multiplier

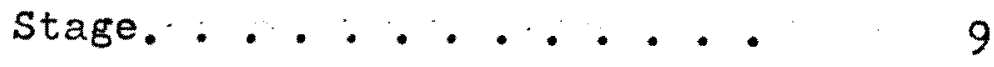

4. Initial Direction of the

Secondary Electrons. . . . ... 9

5 Typical Energy Distribution of

Secondary Electrons. . . . 12

6 Periodicity of Potentials

Along the z-Axis . . . . 17

7 Interpolation of the Potentials. 20

8. Potentials at the Neighborhood

of a Sharp Edge of the

Electrode........ 20

9: Equipotentials for the 10-unit

Spacing Model $(n=23)$..... 23

10 Equipotentials for the 19-unit

Spacing Model $(n=32)$. . . . 24

11. Meridional Trajectory Plots

for the 19-unit spacing

Model for Different Initial

Fositions. . . . . . . . 
12 Meridional Trajectory Plots

for the 10-unit spacing

Model for Different

Initial Energies. . . . .

13 Meridional Trajectory Plots

for the 33-unit spacing

Model for Different

Initial Energies. . . . . .

14 Meridional Trajectory Plots

for the 19-unit spacing

Model for Different

Initial Energies and

Directions. . . . . .

15 Meridional Trajectory Plots

for the 19-unit spacing

Model for Different

Initial Energies and

Directions. . . . . .

16 Meridional Trajectory Plots

for the 10-unit spacing

Model for Different

Initial Positions

and Directions.............. 
17 Skew Trajectory Plots for the

10-unit Spacing Model for

Different Initial

Positions and Directions. ...

18 Skew Trajectory Plots for the

19-unit Spacing lodel for

Different Initial

Directions, . . . . . . .

19 Skew Trajectories Projected onto the XY-plane ...... 


\section{INTRODUCTION}

An electron multiplier is a device by which an input electron current can be multiplied greatly at its output. The ratio of the electron current at the output to that at the input is called the gain of the multiplier. An electron multiplier usually has a number of electrodes, known as dynodes. The surfaces of the dynodes are coated with a material having a high secondary enission coefficient. When primary electrons hit the first dynode with sufficient energy (e.g. 100 electron-volts or more), secondary electrons, whose number may exceed the number of the primaries, are emitted. These electrons, in turn, are accelerated to the second dynode by the voltage applied between the first and second dynodes, where they again release secondaries. If this is continued, the number of secundary electrons at the output of the last dynode can be much greater than the input to the first dynode. An application of the electron multiplier in a cathode ray tube is to improve its performance at high sweeping speed. With increasing sweeping speed in a CRT, the trace on the screen becomes weaker, because 
of the decrease in the electron density on the screen. A multi-channel electron multiplier in front of the screen can increase the electron current density greatly, and hence the brightness of the trace. Such a multiplier consists of a great number of small single channel multipliers as mentioned above. All of the first electrodes of the small multipliers form a honeycomb-like mesh structure in a planar first stage of the multiplier. Similarly, the second stage consists of all of the second dynodes, and so on. Usually there are four to ten stages in such a multi-channel beam current mujtiplier.

In this paper, a multiplier with a conical hole type structure is described. Figure 1(a) shows the structure of a single channel multiplier. The larger side of the hole faces the incoming electrons. The inner walls of the holes are coated with a material having a high coefficient of secondary enission. Figure 1(b) shows a part of the honeycomb structure and Figure $1(c)$ the cross section $S S$ of the structure shown in $1(b)$. The spacing is defined as the distance from the bottom of a stage to the top of the next stage. 

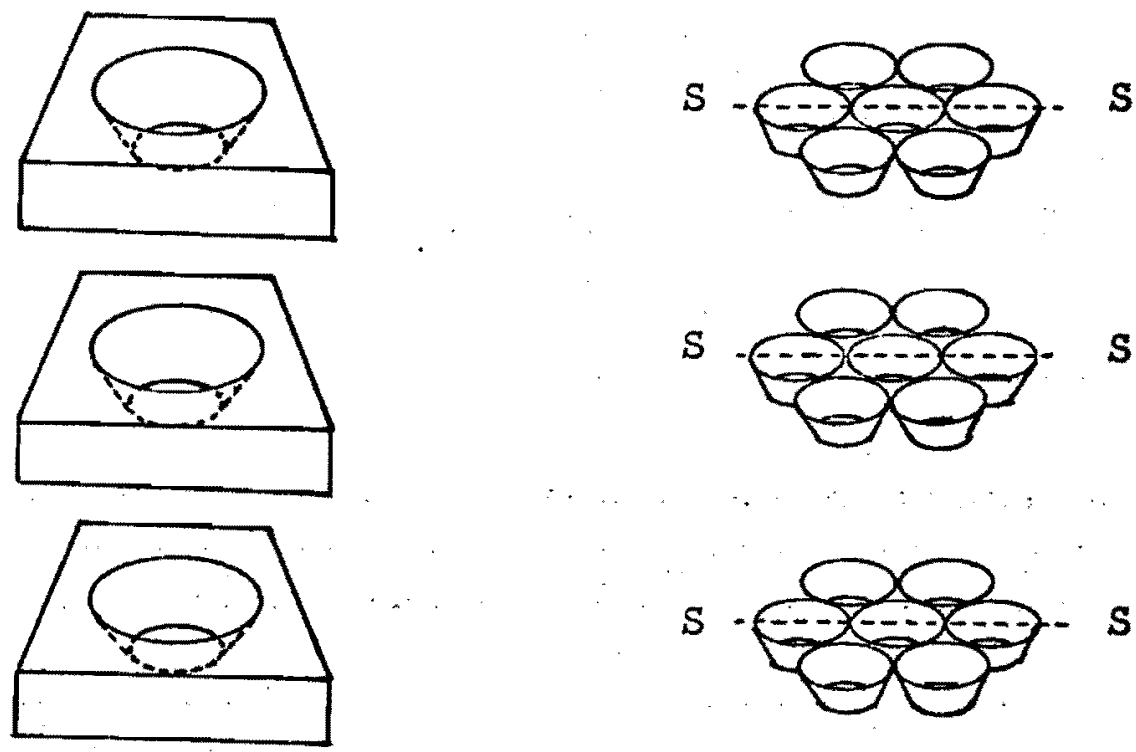

(a) Structure of single channel multiplier.

(b) Honeycomb structure.
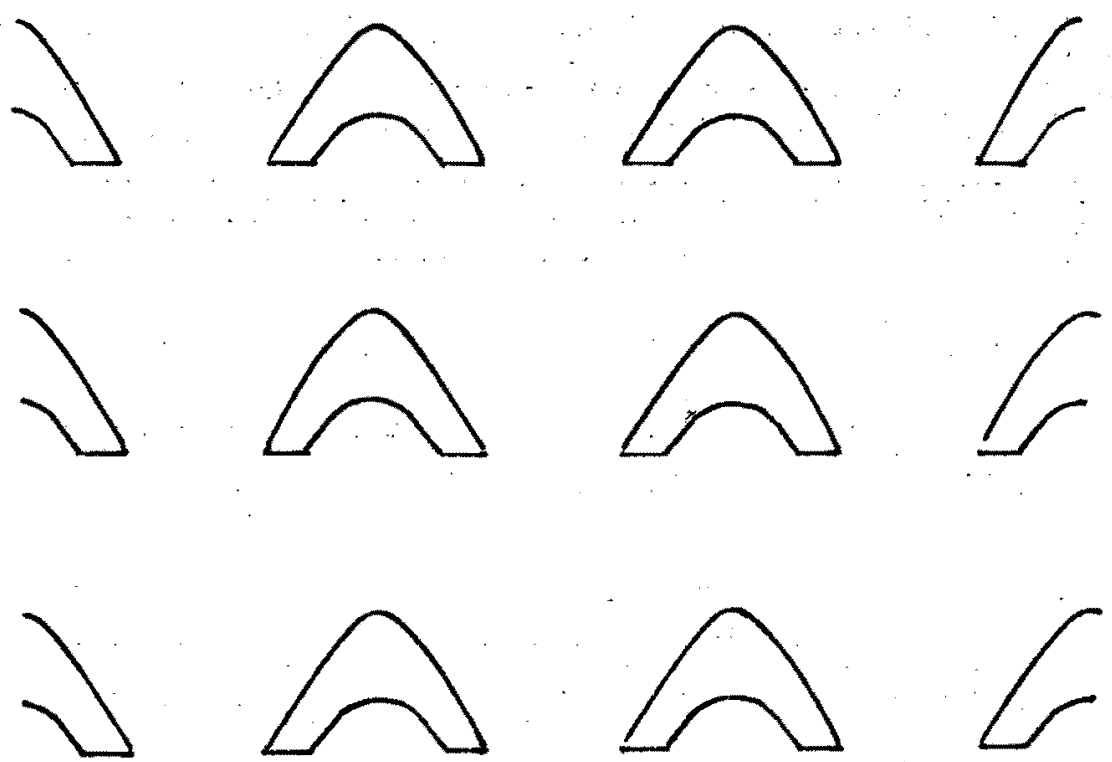

(c) Cross section of the honeycomb structure. Figure 1. Structure of the multiplier 
The main purposes of this paper are: (1) to seek a theoretical basis for the multiplier structure, and (2) to obtain maximum gain by optimizing the spacing between the stages. In order to solve these problems, the potential distribution in the rield and some electron trajectories were calculated. It is essential for the function of the multiplier that most of the secondary electrons enitted from one stage should neither miss the next stage nor hit the original stage, but go from each stage to the next one. To obtain maximum gain, the spacing must be so chosen that most of the secondary electrons from one stage not only go to the next stage but to its active region. The active region is the region on the inner wall surface from which secondary electrons have a good chance to reach the next stage.

The trajectory of an electron depends on the potential distribution and on the initial conditions, i.e. the point of origin and the initial energy and direction of the electron. These factors which influence the trajectories are discussed separately:

1. THE POTENTIAL DISTRIBUTION

The potential distribution derends on the shapes and the potentials of the electrodes. 
In the present study, the only shape parameter varied was the spacing between stages while the shapes of the single stages, shown in Figure 2, were not changed. The electrode dinensions and the spacing are given in terms of an arbitrary length unit $h$, since, for the calculation of the potential distribution and the trajectories, it is not necessary to know the absolute lengths. The potential distribution has been calculated for three different values of the spacing relative to the electrode dimensions.

2. THE POINT OF ORIGIN

The probability of a secondary electron reaching the next stage depends strongly on the position from which it is emitted. Close to the lower edge of the inner wall surface, the electric field pulls the secondaries away from the surface and down through the hole. Farther avay from the edge, the field at the surface has the opposite direction, pushing most of the slow secondaries back to the surface, so that they cannot contribute to the gain. Therefore, the active region is a band 


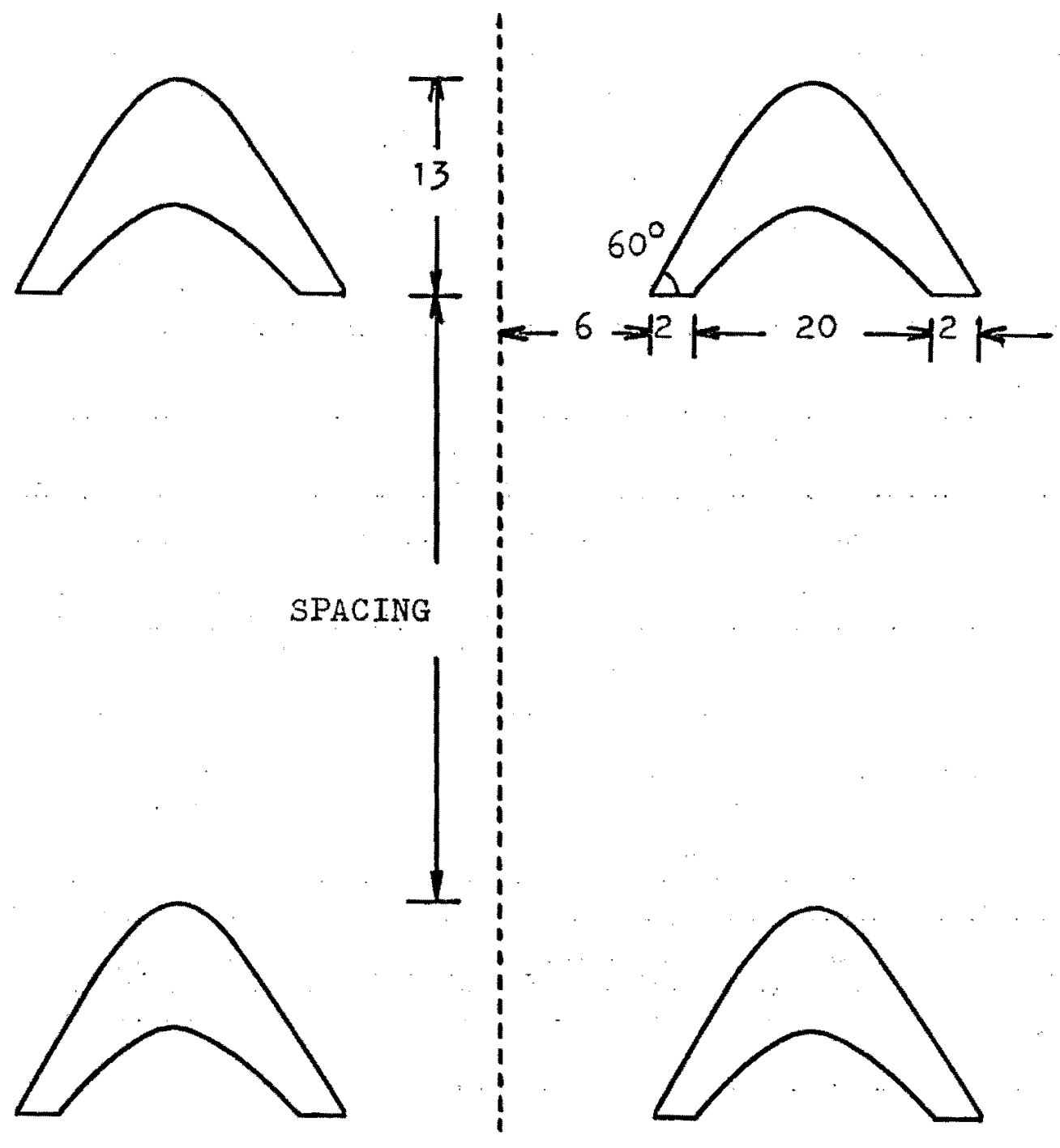

Figure 2. Shape and dimensions of the multiplier. (Unit of length is arbitrary) 
of limited width close to the lower edge, while the local gain in the remaining surface region is negligible. Figure 3 shows qualitatively the dependence of local gain on the radial coordinate in the hole. The equipotential surface which neets the upper stage at right angles, separates the two regions on the inner wall which have different signs of the electric field strength at the surface.

3. THE INITIAL ENERGY OF THE SECONDARY ELECTRONS Electrons with higher initial energies have less curved trajectories than electrons with lower initial energies. In the active region, electrons with all initial energies can leave the surface. In the upper, inactive region, trajectories must have sufficient initial evergy to surpass the energy barrier of the retarding field.

4. THE INITIAL DIRECTION

The initial direction of each secondary electron can be described by two angles $\alpha$ and $\beta . \alpha$ is the angle between the initial direction and the direction of the axis (Figure 
$4(a)) . \quad \beta$ is the angle cetween the projection of the initial direction on a plane perpendicular to the axis and the meridional plane, I.E. the plane through the point of emission and the axis (Figure $4(b)$ ).

Weridional rays $\left(\beta=0^{\circ}\right.$ or $\left.130^{\circ}\right)$ are plotted in the meridional plane. Since a threedimensional ("skew") trajectory in general does not lie in a plane, its geometrical properties cannot be completely represented in a two-dimensional plot. One way of representing it is to show two or more projections, e.g. on the meridional. plane through the axis and the point of origin and on the plane through the axis but perpendicular to the first one, or on a plane perpendicular to the axis. For the present study, it was consiciered sufficient to plot the radial coordinate $r$ as a function of the axial coordinate $z$. It should, however, be borne in mind that a strong curvature in a $r(z)$ plot does not necessarily mean that the trajectory itself is strongly curved. The calculation of the field and of the trajectories is much simplified by the periodicity of the 


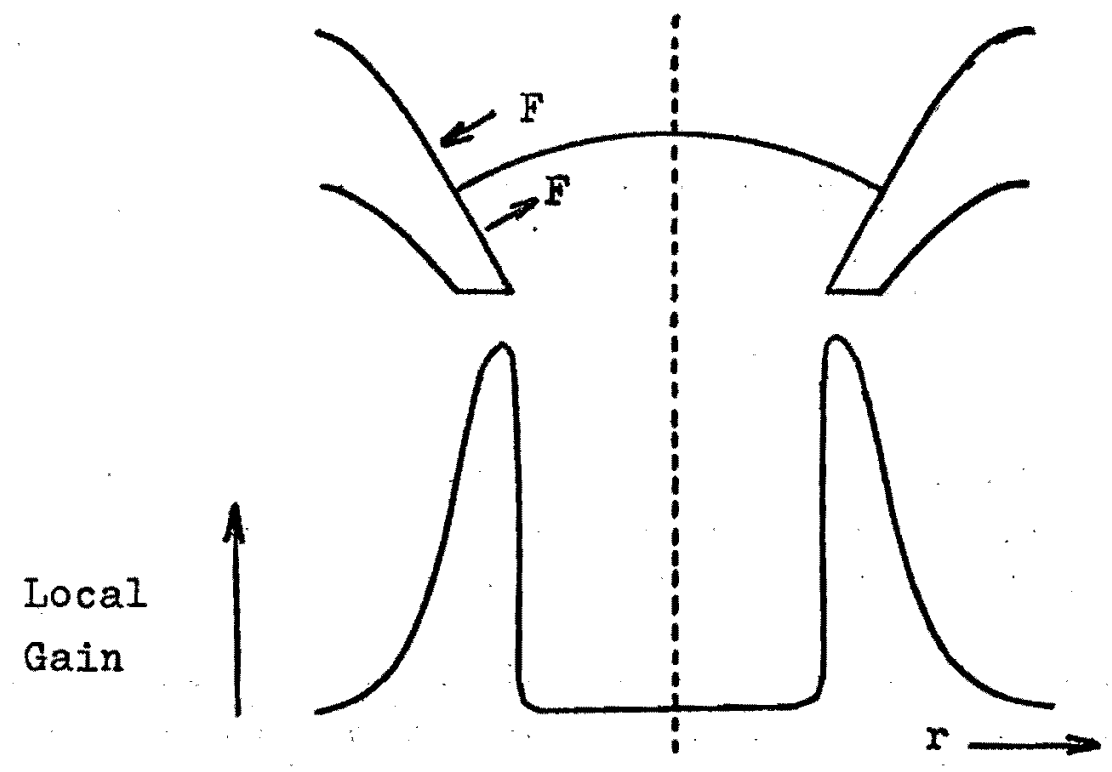

Figure 2. Local gain of the multiplier stage.

Straight line

through point of emission

and parallel to axis
Projection of

initial direction

on plane perpendicular

to axis

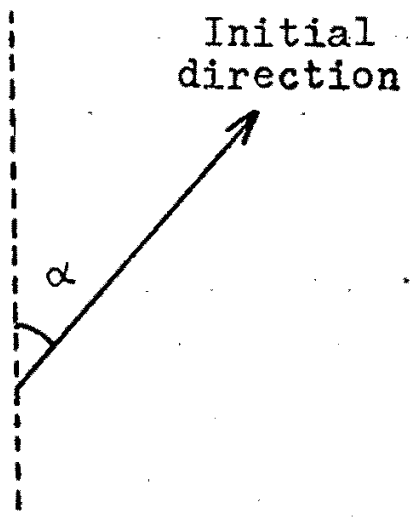

(a) Plane parallel to the axis and containing initial direction.

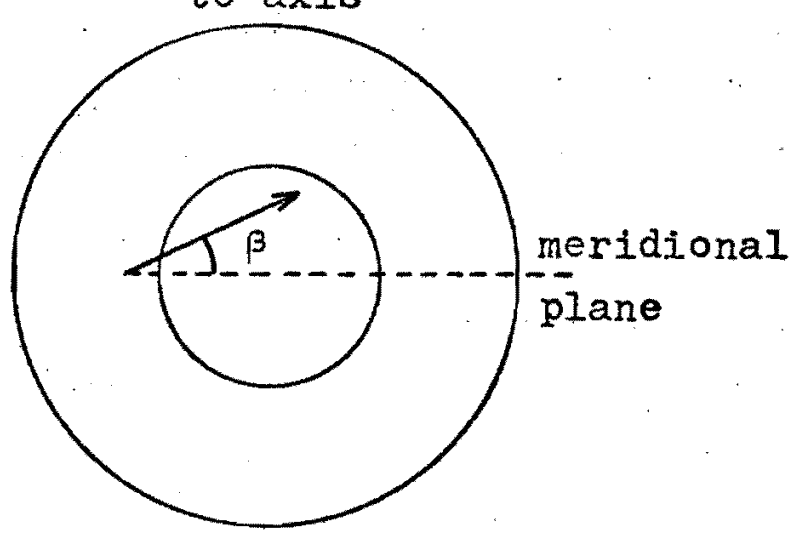

(b) Plane perpendicular to axis.

Figure 4. Parameters and describing the initial direction of the secondary electrons. 
structure. The potential at a point in the $\mathrm{n}$-th stage is equal to the potential of the equivalent point in the $m-t h$ stage plus $(n-m)$ times the potential difference between two subsequent stages. A trajectory of an electron from a point on the surface of the $n$-th stage has the same shape as the trajectory of an electron emitted from the m-th stage with equivalent initial conditions. It is only shifted in the $z$ direction by $(n-m)$ times the periodicity length of the stages in this direction. Corresponding periodicity relations are valid in the sidewise direction so that it is sufficient to study the potential distribution and the trajectories in one periodicity element. Of course, the first and the last stages are exceptions, as far as the periodlcity relations are concerned. 


\section{THE SECONDARY EISSSION FROPERTIES OF THE IRULIPLIER}

The secondary yield $(\delta)$ is defined as the ratio of the averace number of electrons that leave a surface to the number of electrons that bombard it. $\delta$ depends on (1) the material of the surface, (2) the energy of the bombarding (orimary) electrons, and (3) the angle of incidence of the primary electrons.

The secondary electrons have a distribution of initial energy. Figure (5) shows qualitatively this distribution $f(\mathcal{E})$ where $f(\mathcal{E})$ d $\mathcal{E}$ is the fraction of electrons having energies between $\mathcal{E}$ and $\varepsilon+d \varepsilon$. The electrons in the sharp peak at the right side of the energy spectrum are the reflected primaries which have not lost much energy. $\varepsilon_{0}$ is the most probable energy of the prinary electrons. The - electrons corresponding to peak at the left side are the so-called true secondary electrons. They are knocked out by the high velocity prinaries. The distribution has a maximum lying in the range of 2 to 5 electron-volts. The position of the maximum on the energy axis is independent of the energy of the primaries, provided their energy is large compared with the energy spread of the true secondaries. 


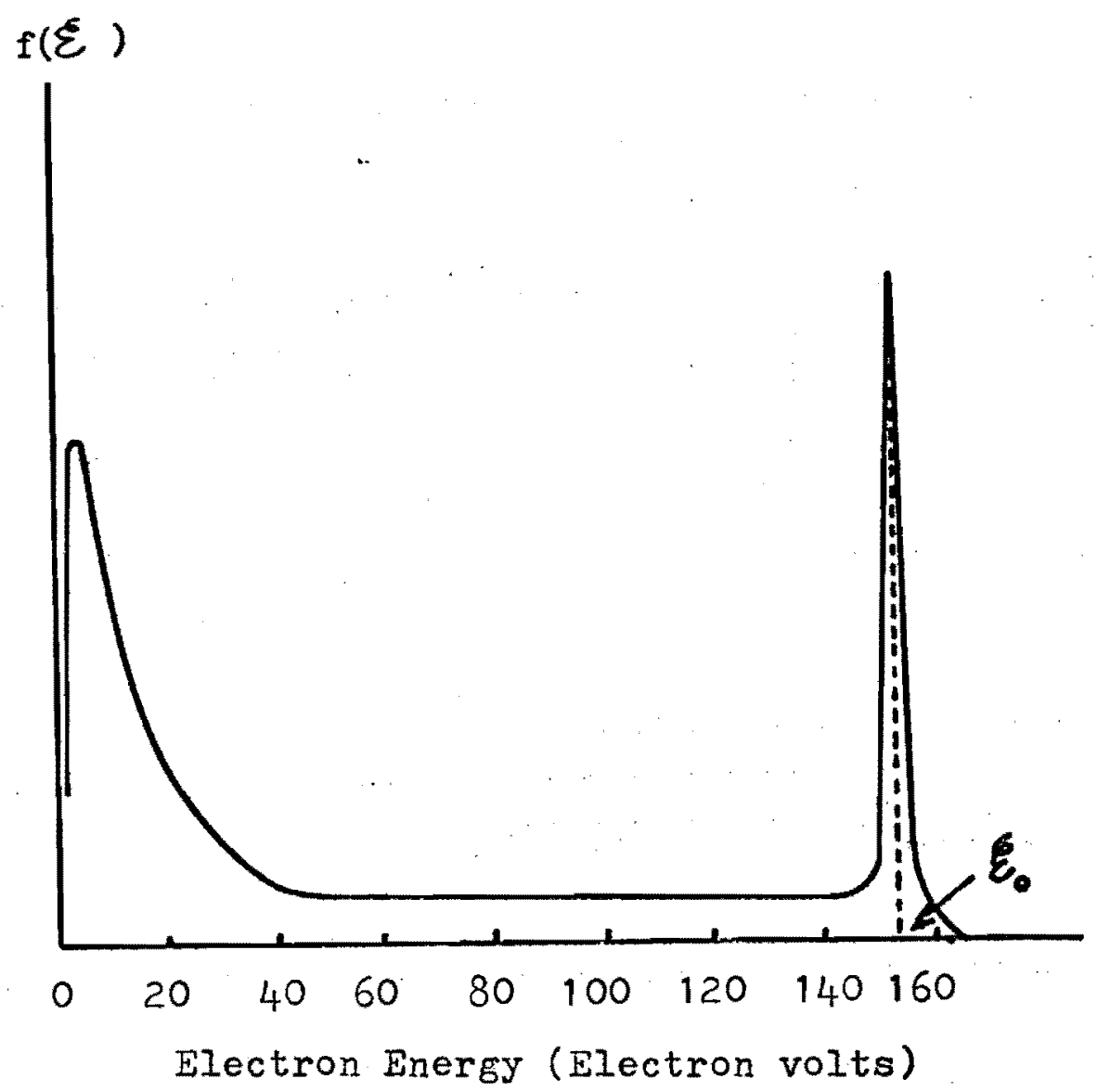

Figure 5. Typical energy distribution of secondary electrons. (Du Mont Multiplier and specification, Second Edition, 1960.) 
The reflected primaries do not contribute much to the function of the electron multiplier because they, as a result of their high energy, travel along almost straight lines between the stages and are hardly bent by the electric field between the stages. Therefore, our attention will be directed to the true secondary electrons. Furthermore, we are most inter- ested in the electrons emitted normal to the surface, because the number of electrons emitted in a direction making an angle $\theta$ with the normal is proportional to $\cos \theta$. 


\section{THE DETERAINATION OF THE POTENTAL DISPRIBUTION}

Since neither the potential distribution nor the trajectories can be written in the forn of simple mathematical functions, numerical method nust be employed.

Liebnann's numerical method (Southwell 1956) of calculating the potential distribution in an electrostatic field consists of replacing the derivatives in Laplace's equation by finite differences of the potentials at a great number of eouidistant mesh points in a quadratic mesh system stretched over the field. Relations between the potential at each nesh point and the potential at its neighboring mesh points are used to perforn successive iterations converging towards a final solution wich approxinates the potential distribution. The choice of mesh size depends on the fineness of detail of the potential distribution needed. The smaller the size, the more accurate the distribution. However, snaller mesh size means a greater number of nesh points, and hence more potential values to be determined. This also means that more time is needed for the calculation. The number of points is inversely proportional to the square of the mesh size, and the nuinber of iterations required increases even faster. To compensate for this drawback, a practical method is to have a smaller nesil size in the regions of strong field variation and a 
larger one in the regions with a more hoogeneous field. Une of the three potential distributions calculated in this paper (the 33-unit spacing, see Appendix D) was done in this way with a desk calculator.

However, if the calculation is to be done by a computer, the mixed mesh size arrangement needs more programing time than a smaller uniform mesh system. When this is weighed against the actual computer time, the smaller uniform nesh system is less costly. The other two calculations of the potential distribution were done by this method (Anpendices $B$ and $C$ ). The arbitrary length unit mentioned on page 4 was chosen equal to the mesh size in this case.

The numerical method used is based on Laplace's equation in cylindrical coordinates (Becker 1954), which in this case of rotational symmetry is:

$$
\frac{\partial^{2} \phi}{\partial r^{2}}+\frac{1}{r} \frac{\partial \phi}{\partial r}+\frac{\partial^{2} \phi}{\partial z^{2}}=0
$$

For the different cases listed below, Liebmann's procedure yields the following finite difference formulas with mesh size h:

1. In general, except for the special categories

$$
\begin{aligned}
& \text { following, } \\
& \begin{aligned}
\phi(r, z)= & \frac{1}{4}[\phi(r+h, z)+\phi(r-h, z)+\phi(r, z+h)+\phi(r, z-h)] \\
& +\frac{h}{8 r}[\phi(r+h, z)-\phi(r-h, z)]
\end{aligned}
\end{aligned}
$$


2. On the axis

$$
\phi(0, z)=\frac{1}{6}[4 \phi(h, z)+\phi(0, z+h)+\phi(0, z-h)]
$$

3. On the outer boundary with respect to $r$ if the equipotentials are perpendicular to the boundary $\left(\frac{\partial \phi}{\partial r}=0\right)$

$$
\phi(r, z)=\frac{1}{4}[2 \phi(r-h, z)+\phi(r, z+h)+\phi(r, z-h)]
$$

4. At the boundaries in the z-direction, formula (2) cannot be applied immediately because no potential values are available at mesh points beyond the boundaries. If there is a total of $n$ meshes along the $\mathrm{z}$-axis in one of the regions as shown in Figure $1(c)$, then from Figure 6 , the potential at $z=-h$ differs from that at $z=(n-1) h$ by the constant accelerating voltage $\phi_{0}$ between the successive stages. Thus we have

$$
\phi(r,-h)=\phi(r,(n-1) h)-\phi_{0}
$$

Correspondingly, we have

$$
\begin{aligned}
& \phi(r,-2 h)=\phi(r,(n-2) h)-\phi_{0} \\
& \phi(r,(n+1) h)=\phi(r, h)+\phi_{0}
\end{aligned}
$$

Equations (5) to (7) are used to supply the potentials at the mesh points beyond the boundary."

* This method of replacing boundary conditions by a condition of periodicity has not to our knowledge been described elsewhere. 

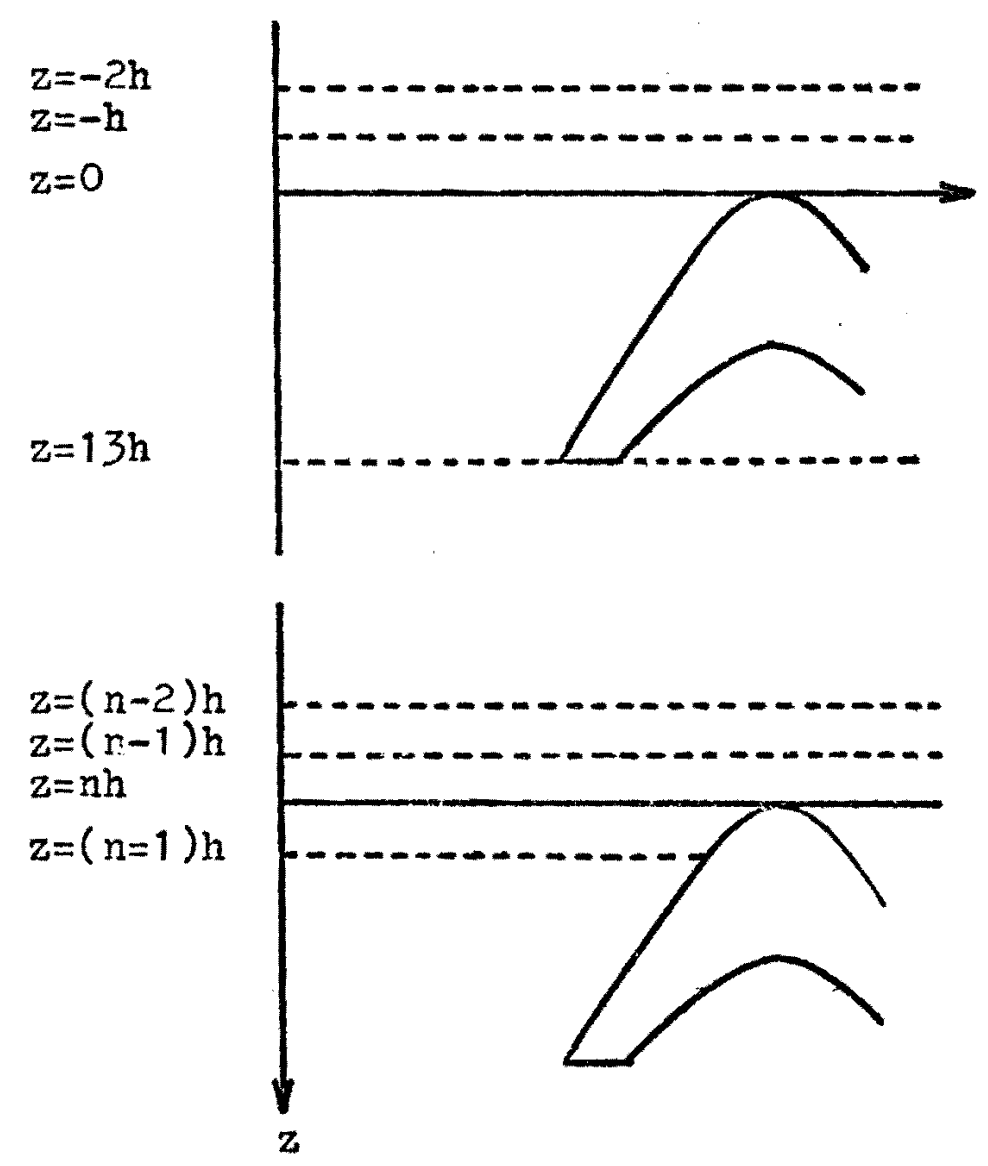

z-axis.

Figure 6. Periodicity of potential along 
5. When the electrode does not intersect the mesh lines in integer mesh points, formula (2) cannot be applied at points close to the electrode. The potentials at such points are determined by interpolation. The potential at a point a distance ah $(0<a<1)$ from the electrode along the r-axis is determined using a three-point Lagrange inter-... polation formula (Abramowitz, et al., 1964) (Figure 7).

$$
\phi=\frac{2}{(1+a)(2+a)} \phi_{c}+\frac{2 a}{1+a} \phi_{1}-\frac{a}{2+a} \phi_{2}
$$

As a special case, if $\phi_{c}=0,(8)$ becomes

$$
\phi=\frac{2 a}{1+a} \phi_{1}-\frac{a}{2+a} \phi_{2}
$$

This is the interpolation formula used in this paper, and it can be applied to either the $r$ or the $z$ coordinate. In case the potential at a point can be found by applying interpolation to either the $r$ or the $z$ coordinate, the coordinate corresponding to the smaller "a" value is chosen.

6. The potentials at points close to a sharp edge of the electrode, e.g. the bottom rim in our case, should not be calculated by one of the foregoing formulas. This is because, at such a sharp edge, 
the field strength becomes infinite while the potential remains finite. Assume that $\boldsymbol{f}$ is the angle (in radians) of the sharp edge and that this edge coincides with an integer mesh point $(r, z)$, as shown in Figure 8. An analysis from a solution of Laplace's equation gives (Lenz 1968) $\phi(r-h, z)=\left(\frac{1}{2}\right)^{\pi /(2 \pi-\gamma)} \phi(r-2 h, z)-\left[1-\left(\frac{1}{2}\right)^{\pi /(2 \pi-\gamma)}\right] \phi(r, z) \cdots$ In our case, $\gamma=\frac{\pi}{3}$, we have

$$
\frac{\pi}{2 \pi-\gamma}=\frac{3}{5}=0.6
$$

and

$$
\begin{aligned}
\phi(r-h, z) & =\left(\frac{1}{2}\right)^{0.6} \phi(r-2 h, z)+\left[1-\left(\frac{1}{2}\right)^{0.6}\right] \phi(r, z) \\
& =0.6598 \phi(r-2 h, z)+0.3402 \phi(r, z)
\end{aligned}
$$

If $\phi(r, z)=0$, we have

$$
\phi(r-h, z)=0.6598 \phi(r-2 h, z)
$$

Similarly, for the point which is one mesh space from the sharp edge along the z-axis, we have

$$
\phi(r, z+h)=0.6598 \phi(r, z+2 h)
$$

The formulas (2) through (11) are used for the calculation of the potentials at the integer mesh points. Each of them applies to a different type of location. 


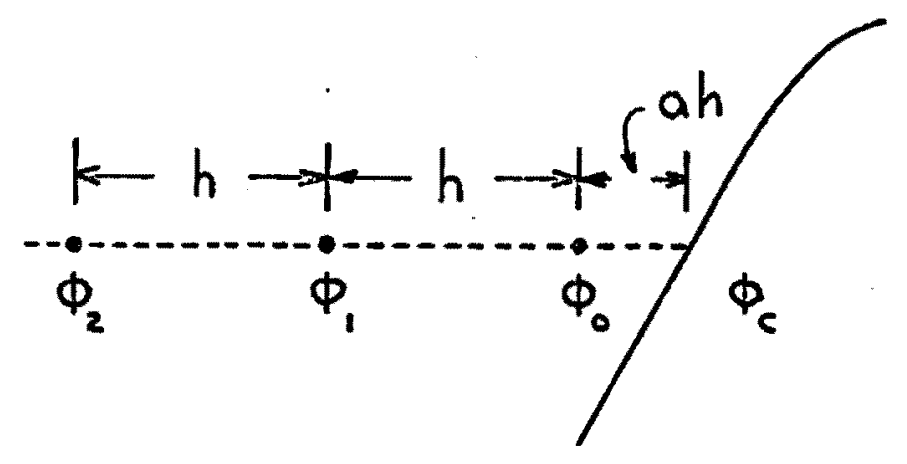

Figure 2. Interpolation of the potentials.

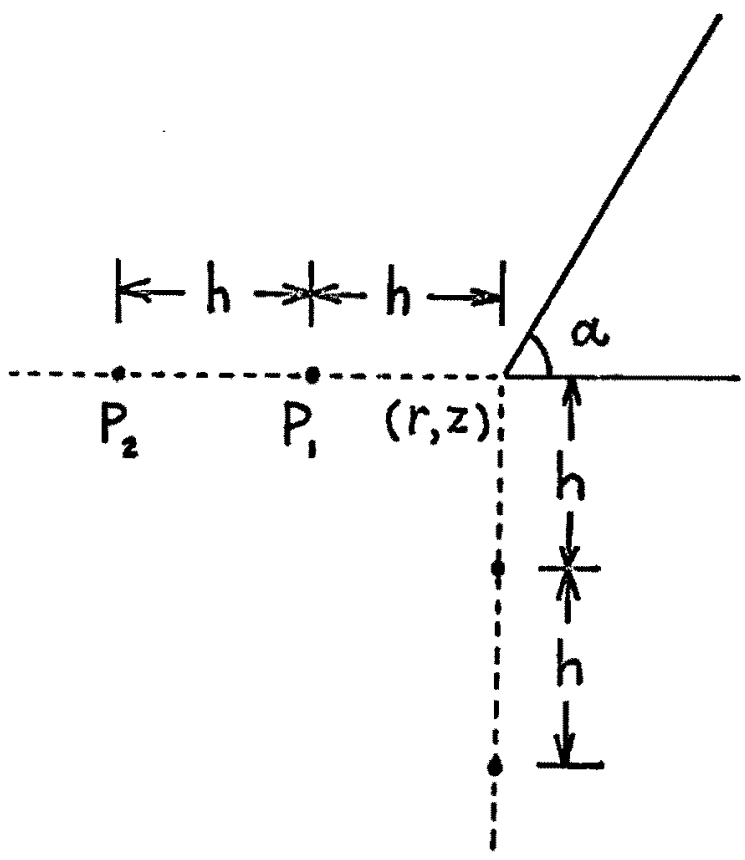

Figure 8. Potentials at the neighborhood of a sharp edge of the electrode. 
A crude guess of the potential distribution is used to obtain the next approximation, and so on. This iterative rrocedure is continued until the greatest change of any potential value per iteration becomes small compared with the desired accuracy.

The order in which the new potentials in the different mesh points are calculated has no effect on the result, but each mesh point should be treated in each iteration. In this computation, the mesh points were scanned radially outward starting from the z-axis. An entire sweep over the field fron $z=n$ to $z=0$ constitute one iteration. Alnost any initial approximation satisfying the boundary condition can be used because the potentials converge during the calculation. However, a good guess reduces the calculation time. Starting from a rough approximation, usually between two and three hundred iterations were needed to obtain an accuracy of the order of $0.01 \%$.

In this paper, the initial potentials at the integer mesh points in the space from $z=0$ to $z=13$ were assumed to be zero, the potential of the electrode. In the space from $z=13^{\circ}$ to $z=n$, the potential was set equal to

$$
\frac{z-13}{n-13} \phi_{0}
$$

i.e. a homogenious field was assumed. 
Because of the linearity of Laplace's equation, the potentials at the upper and at the lower electrodes of a stage may be chosen arbitrarily. For convenience, the calculation was performed with a potential value of zero for the upper and 10,000 units for the lower electrode. If the corresponding solution is $\phi(r, z)$, then a solution, where the upper stage is at $V$ and the lower at $\mathrm{V}+\phi_{0}$, is

$$
V+\phi_{0} \frac{\phi(r, z)}{10,000}
$$

The potential distribution $\phi(r, z)$ was calculated for the cases $n=23, n=32$, and $n=46$ corresponding to spacings 10, 19, and 33, respectively. Appendix A contains the computer (IBM 1130) program used for the calculation of the potential distribution. Appendices $B, C$, and $D$ are the final converged distributions for 10-, 19-, and 33-unit spacings, and Figures 9 and 10 show the equipotential plots for the cases of 10-unit $(n=23)$ and 19-unit ( $n=32)$ spacings. 


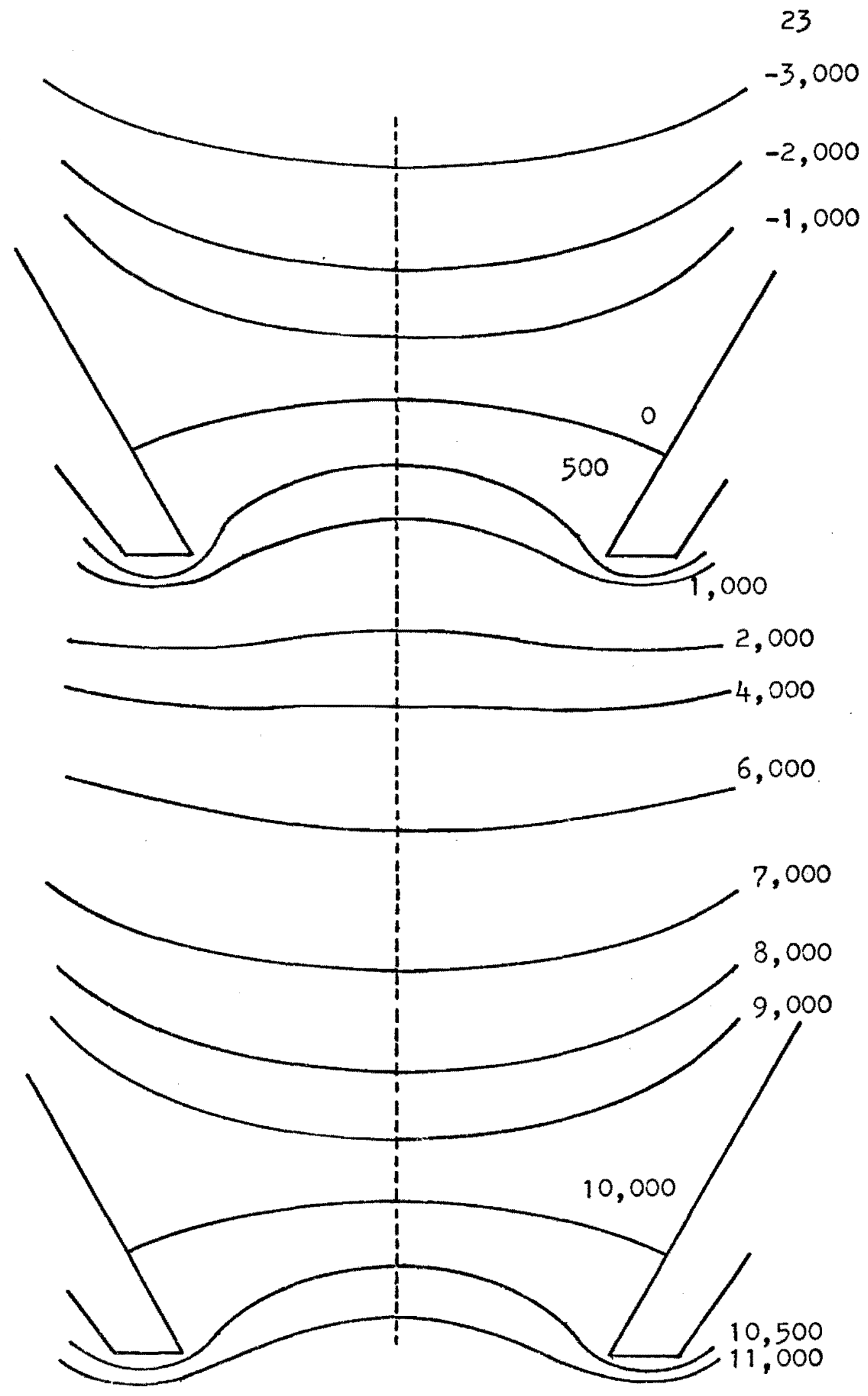

Figure 2. Equipotentials for 10-unit spacing $(n=23)$ 


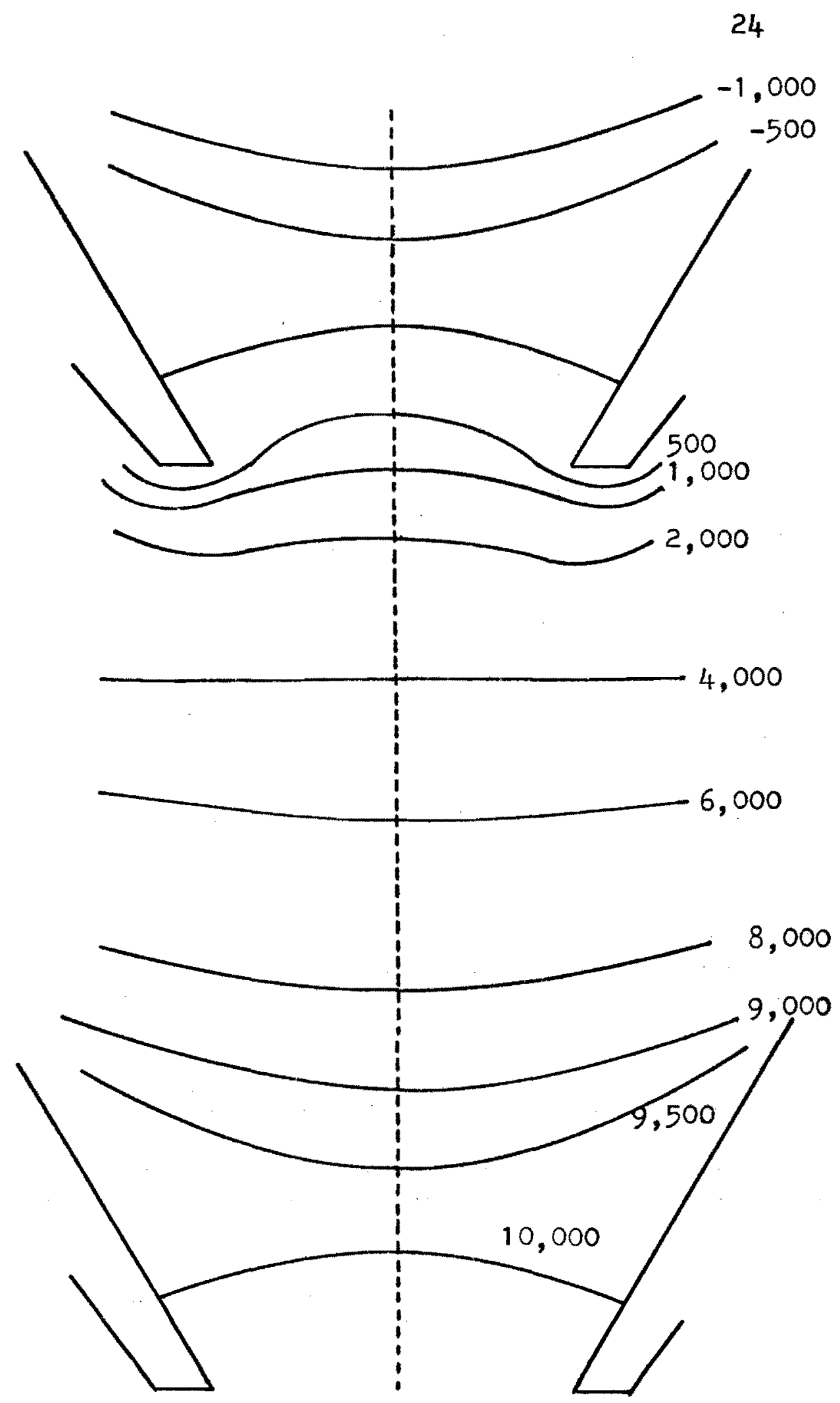

$(n=32)$.

Figure 10. Equipotentials for 19-unit spacing 
The equation of motion of non-relativistic electrons in an electrostatic field is

$$
m \frac{d^{2} \vec{r}}{d t^{2}}=e \nabla \phi(\vec{r})
$$

where $m$ and $e$ are the mass and charge of the electron, respectively. As our interest is in the geometrical properties of the trajectories and not the dependence of the trajectory coordinates on time $t$, we may eliminate the operator $\frac{d}{d t}$ by using

$$
v \frac{d}{d s}=\frac{d}{d t}
$$

where $V$ is the velocity of the electron and $s$ the path length. Substituting into (12), we have

$$
m v \frac{d}{d s}\left(v \frac{d \vec{r}}{d s}\right)=e \nabla \phi(\vec{r})
$$

In this non-relativistic problem, we have

$$
V=\sqrt{\frac{2 e}{m}(\phi+E)}
$$

where $e E$ is the total energy of the electron. Substituting into (13), we get

$$
\sqrt{2(\phi+E)} \frac{d}{d s}\left(\sqrt{2(\phi+E)} \frac{d \vec{r}}{d s}\right)=\nabla \phi(\vec{r})
$$

If this vector equation is written in Cartesian components, we have 


$$
\begin{aligned}
& 2 \sqrt{\phi+E} \frac{d}{d s}\left(\sqrt{\phi+E} \frac{d x}{d s}\right)=\frac{\partial \phi}{\partial x} \\
& 2 \sqrt{\phi+E} \frac{d}{d s}\left(\sqrt{\phi+E} \frac{d y}{d s}\right)=\frac{\partial \phi}{\partial y} \\
& 2 \sqrt{\phi+E} \frac{d}{d s}\left(\sqrt{\phi+E} \frac{d z}{d s}\right)=\frac{\partial \phi}{\partial z}
\end{aligned}
$$

In our cylindrically symmetrical problem, $\phi$ does not depend on the azimuth angle $\psi$. Thus

$$
\frac{\partial \phi}{\partial x}=\frac{\partial \phi}{\partial r} \frac{\partial r}{\partial x}=\frac{x}{r} \frac{\partial \phi}{\partial r}=\frac{x}{\sqrt{x^{2}+y^{2}}} \frac{\partial \phi}{\partial r}
$$

Similarly,

$$
\frac{\partial \phi}{\partial y}=\frac{\partial \phi}{\partial r} \frac{\partial r}{\partial y}=\frac{y}{r} \frac{\partial \phi}{\partial r}=\frac{y}{\sqrt{x^{2}+y^{2}}} \frac{\partial \phi}{\partial r}
$$

Substituting into (15), we get

$$
\begin{aligned}
& 2 \sqrt{\phi+E} \frac{d}{d s}\left(\sqrt{\phi+E} \frac{d x}{d s}\right)=\frac{x}{\sqrt{x^{2}+y^{2}}} \frac{\partial \phi}{\partial r} \\
& 2 \sqrt{\phi+E} \frac{d}{d s}\left(\sqrt{\phi+E} \frac{d y}{d s}\right)=\frac{y}{\sqrt{x^{2}+y^{2}}} \frac{\partial \phi}{\partial r} \\
& 2 \sqrt{\phi+E} \frac{d}{d s}\left(\sqrt{\phi+E} \frac{d z}{d s}\right)=\frac{\partial \phi}{\partial z}
\end{aligned}
$$

This system of three ordinary non-linear second-order differential equations can be transformed into a system of six ordinary non-linear first order differential equations: 


$$
\begin{aligned}
& \frac{d x}{d s}=\frac{1}{\sqrt{\phi+E}} p ; \quad \frac{d p}{d s}=\frac{1}{\sqrt[2]{\phi+E}} \frac{x}{\sqrt{x^{2}+y^{2}}} \frac{\partial \phi}{\partial r} \\
& \frac{d y}{d s}=\frac{1}{\sqrt{\phi+E}} q ; \quad \frac{d q}{d s}=\frac{1}{\sqrt{\phi+E}} \frac{y}{\sqrt{x^{2}+y^{2}}} \frac{\partial \phi}{\partial r} \\
& \frac{d z}{d s}=\frac{1}{\sqrt{\phi+E}} u ; \quad \frac{d u}{d s}=\frac{1}{2 \sqrt{\phi+E}} \frac{\partial \phi}{\partial z}
\end{aligned}
$$

From here, the trajectories were computed by a Fortran computer program using the Runge-Kutta procedure (Lenz 1970). In this program, the initial Cartesian coordinates $x, y$, and $z$, the total energy, and the two parameters $\alpha$ and $\beta$ (Figure 4) defining the initial direction are input. The step width along the trajectory is also input. It can be changed during a trajectory run. The potential distributions were supplied by the computer program described in Appendix A.

The printout of the result of the calculation of the trajectories from the computer contains a set of four coordinates, $\mathrm{x}, \mathrm{y}, \mathrm{z}$, and $\mathrm{r}$. The meridional trajectories for which $\mathrm{y}=0$ are plotted in the $\mathrm{xz}$-plane. The skew trajectories are plotted in cylindrical coordinates $r$ and $z$. Figures 11 to 19 show some trajectories for spacings of 10, 19, and 33 mesh units.

Figure 11 shows the meridional trajectories in 
a model with a spacing between stages of 19 mesh units. The secondary electrons were assumed to have initial energies of $3 \%$ of the accelerating voltage and initial directions normal to the surface. This plot shows the effect of the emitting position on the trajectories. The closer the enitting point to the edge, the farther from the edge of the next stage is the landing point of the trajectory. Trajectory 4 misses the next stage and trajectory 5 returns back to the original stage.

Figure 12 and 13 show trajectories with different initial energies, initially normal to the surface. In the 33-unit spacing nodel, Figure 13, the trajectories with $4 \%$ and $5 \%$ initial energies went to the opposite wall of the original stage. Only electrons with energies less than somewhere between $3 \%$ and $4 \%$ could go to the next stage. However, in Figure 12, a plot of the 10-unit spacing model, electrons with initial energies up to much more than $4 \%$ go to the next stage. It appears that only electrons with very high initial energies would be stopped by the original stage, but their nuinber can be assumed to be very small. Trajectories for the 19-unit spacing model are shown in Figure 14. 
Trajectories 1 and 3 which are initially normal to the surface can be compared with the trajectories of the 10-unit spacing model. They land in a less sensitive region farther from the edge of the next stage. Thus, as far as the secondary electron gain is concerned, the 10-unit spacing model is the best among the three. The trajectories 2, 3, and 4 in Figure 14 are meridionals in the 19-unit spacing model with the same initial energy of $3 \%$, but with different values of the angle $\alpha$. The trajectories with different $\alpha$ values cross each other, forming an envelope. An envelope is also formed by trajectories 2, 3, and 4 in Figure 15, which are emitted from a different point $\left(r_{0}=8\right)$. Figure 16 shows some trajectories for the 10-unit spacing model. The trajectories 1 and 2 are meridionals emitted from point $r_{0}=7$, while 3 and 4 are from the point $r_{0}=8$. Again, trajectories emitted at the same point with the same energy but different $\alpha$ values form an envelope.

Figures 17 and 18 show some skew trajectories in the coordinates $r$ and $z$. Fjgure 17 is for 10-unit spacing, and Figure 18 is for 19-unit spacing. As $r$ equals $\sqrt{x^{2}+y^{2}}$, it has always positive sign though $x$ and/or y may change sign. The curves in these graphs 
show radial distances from the axis. Figure 19 is

a projection of the three trajectories of Figure 18 on the $\mathrm{xy}$-plane. 


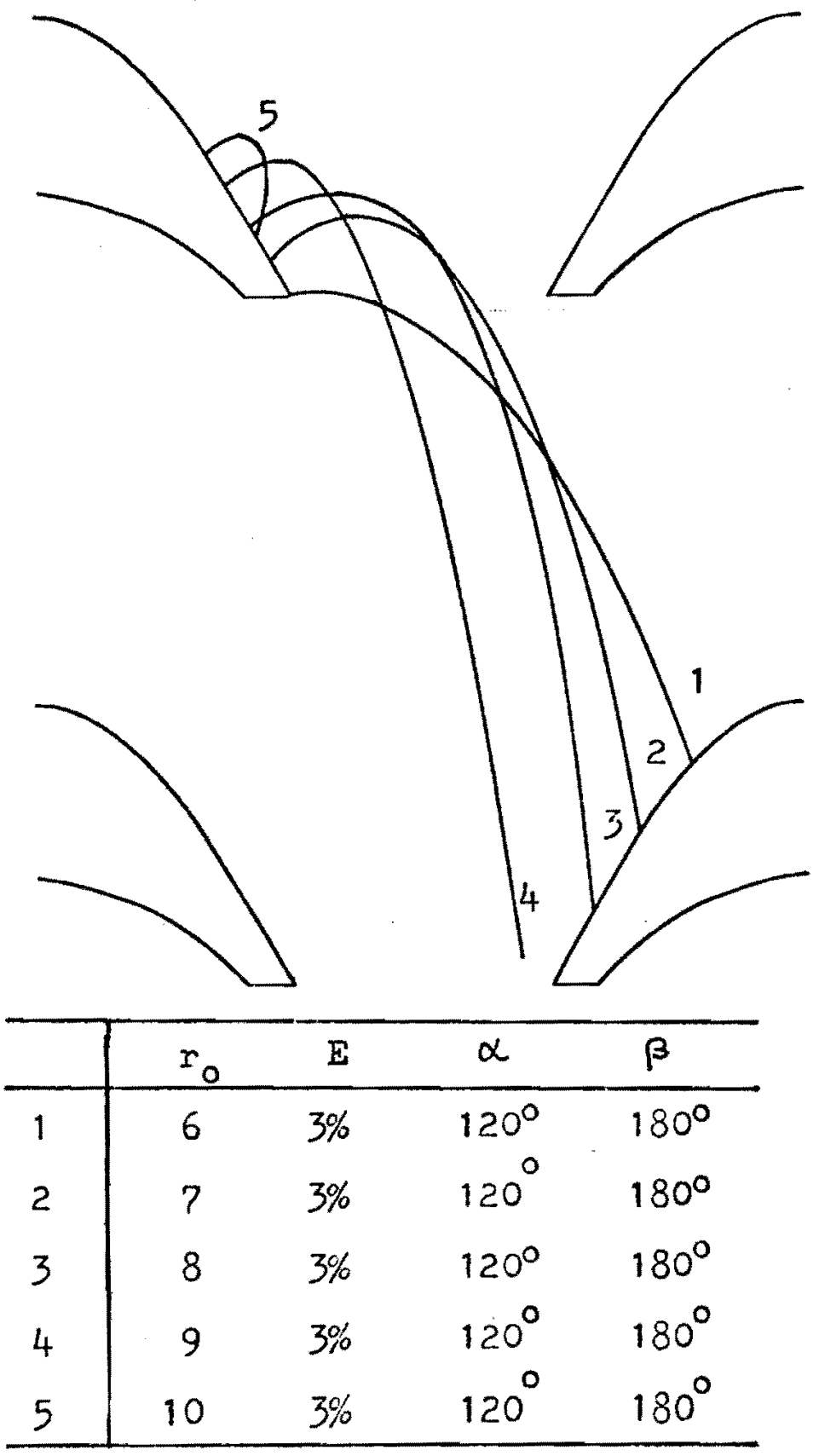

Figure 11. Meridional trajectory plots for the 19-unit spacing model for different initial positions. 

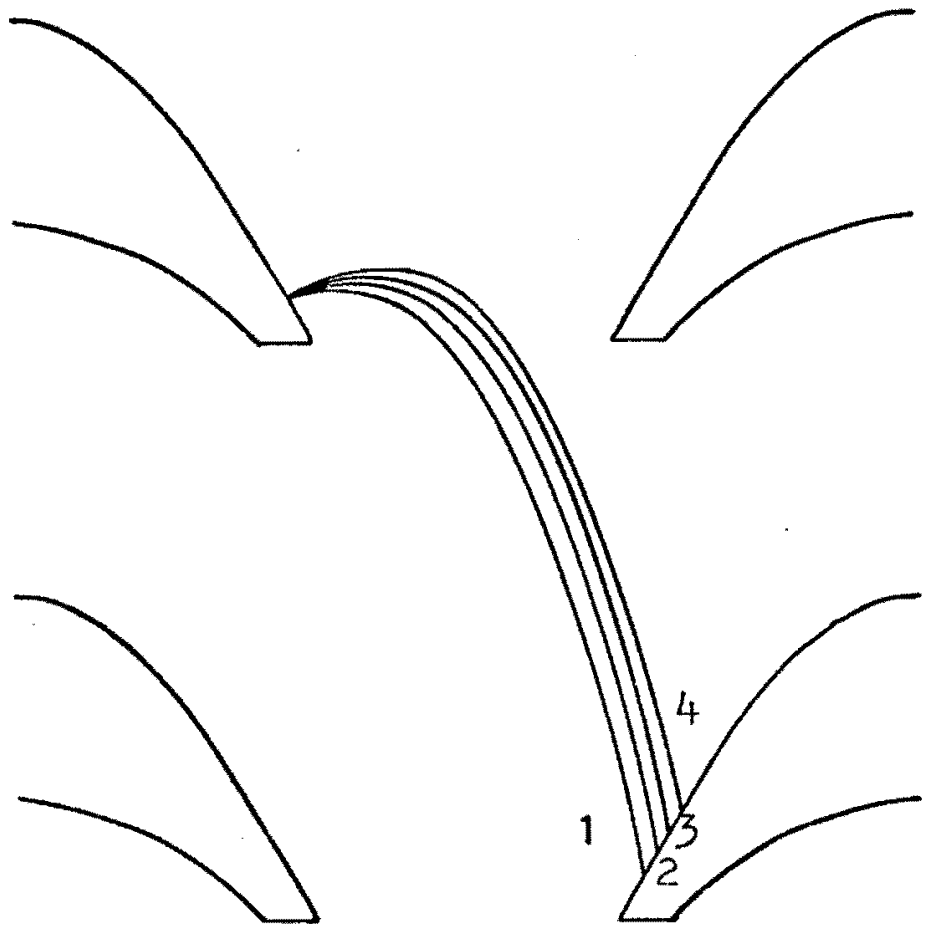

\begin{tabular}{c|cccc}
\hline & $\mathrm{r}_{\mathrm{o}}$ & $\mathrm{E}$ & $\alpha$ & $\beta$ \\
\hline 1 & 7 & $1 \%$ & $120^{\circ}$ & $180^{\circ}$ \\
2 & 7 & $2 \%$ & $120^{\circ}$ & $180^{\circ}$ \\
3 & 7 & $3 \%$ & $120^{\circ}$ & $180^{\circ}$ \\
4 & 7 & $4 \%$ & $120^{\circ}$ & $180^{\circ}$ \\
\hline
\end{tabular}

Figure 12. Meridional trajectory plots for the 10-unit spacing model for different initial positions. 

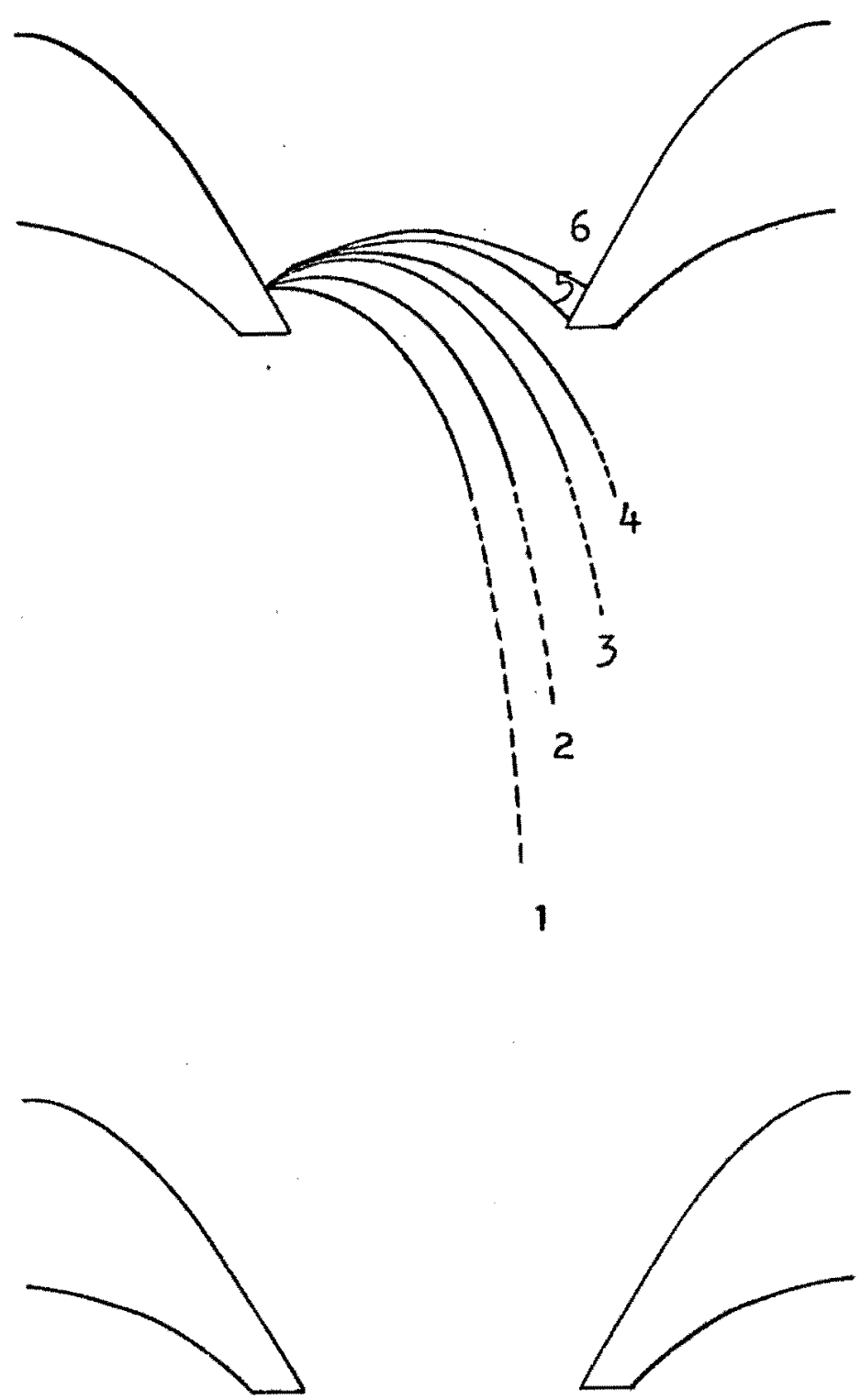

\begin{tabular}{c|cccc}
\hline & $r_{0}$ & $E$ & $\alpha$ & $\beta$ \\
\hline 1 & 7 & 0 & $120^{\circ}$ & $180^{\circ}$ \\
2 & 7 & $1 \%$ & $120^{\circ}$ & $180^{\circ}$ \\
3 & 7 & $2 \%$ & $120^{\circ}$ & $180^{\circ}$ \\
4 & 7 & $3 \%$ & $120^{\circ}$ & $180^{\circ}$ \\
5 & 7 & $4 \%$ & $120^{\circ}$ & $180^{\circ}$ \\
6 & 7 & $5 \%$ & $120^{\circ}$ & $180^{\circ}$ \\
\hline
\end{tabular}

Figure 13. Meridional trajectory plots for the 33-unit spacing model for different initjal energies. 

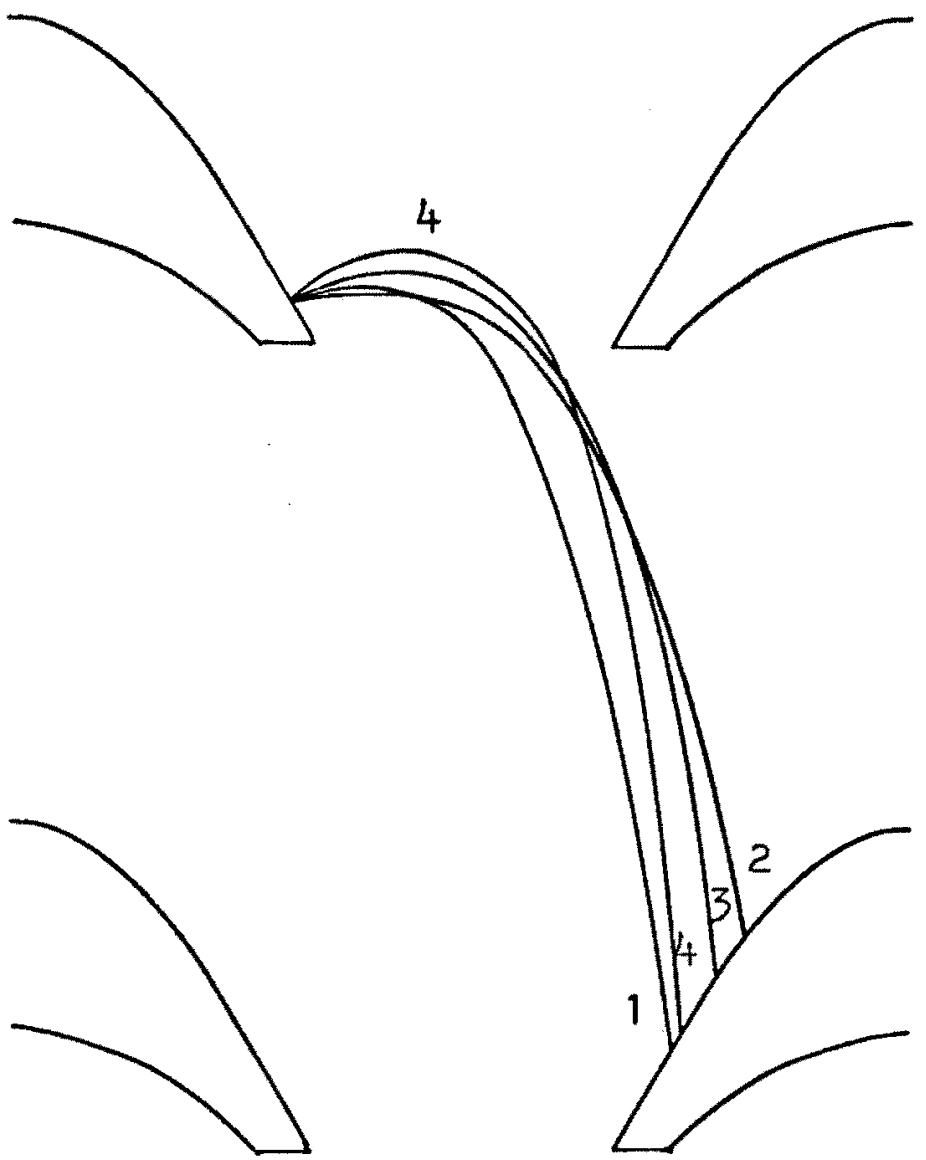

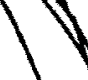

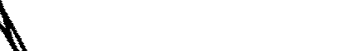

\begin{tabular}{c|cccc}
\hline & $r_{0}$ & $\mathrm{E}$ & $\alpha$ & $\beta$ \\
\hline 1 & 7 & $1 \%$ & $120^{\circ}$ & $180^{\circ}$ \\
2 & 7 & $3 \%$ & $110^{\circ}$ & $180^{\circ}$ \\
3 & 7 & $3 \%$ & $120^{\circ}$ & $180^{\circ}$ \\
4 & 7 & $3 \%$ & $130^{\circ}$ & $180^{\circ}$ \\
\hline
\end{tabular}

Figure 14. Meridional trajectory plots for the 19-unit spacing model for different initial energies and directions. 


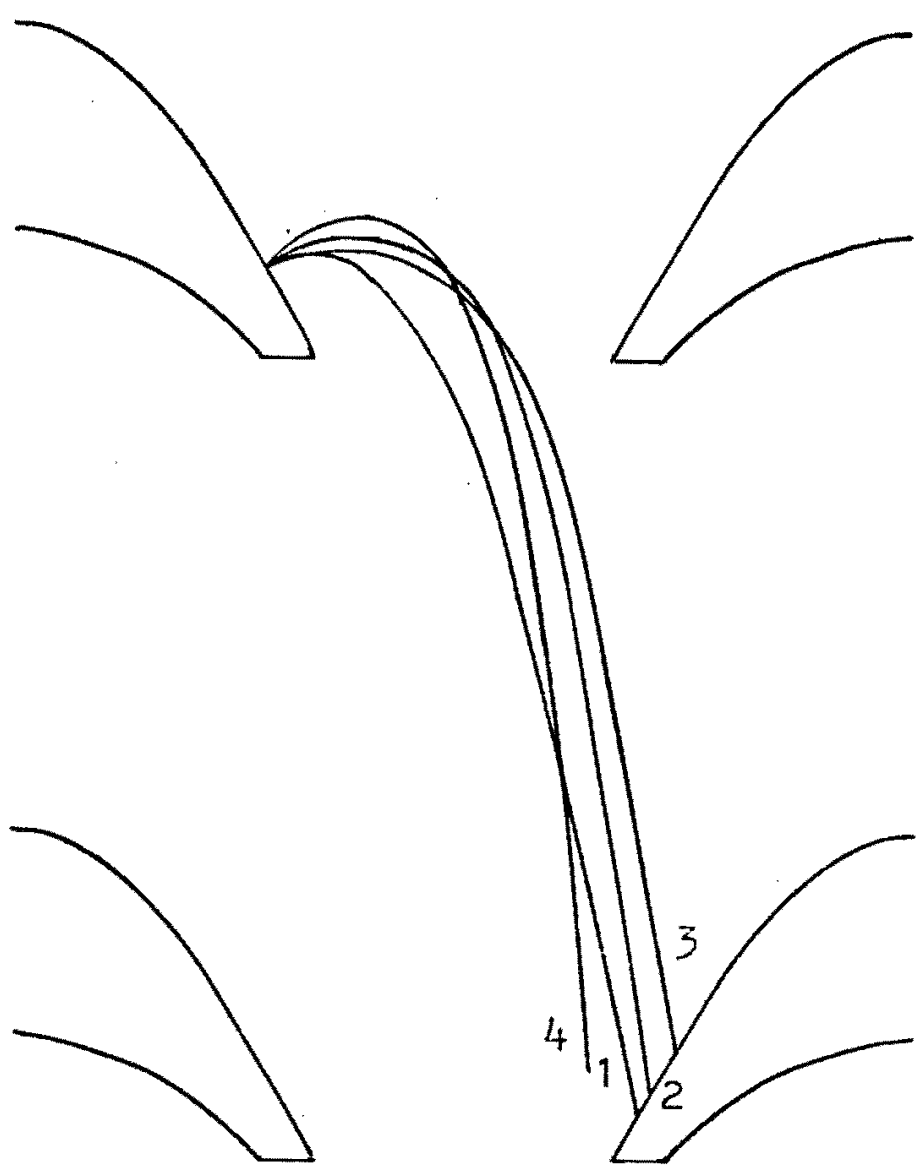

\begin{tabular}{c|cccc}
\hline & $\mathrm{r}_{\mathrm{O}}$ & $\mathrm{E}$ & $\alpha$ & $\beta$ \\
\hline 1 & 8 & $1 \%$ & $120^{\circ}$ & $180^{\circ}$ \\
2 & 8 & $3 \%$ & $120^{\circ}$ & $180^{\circ}$ \\
3 & 8 & $3 \%$ & $110^{\circ}$ & $180^{\circ}$ \\
4 & 8 & $3 \%$ & $130^{\circ}$ & $180^{\circ}$ \\
\hline
\end{tabular}

Fioure 15. Meridional trajectory plots for the 19-unit spacing model for different initial energies and directions. 


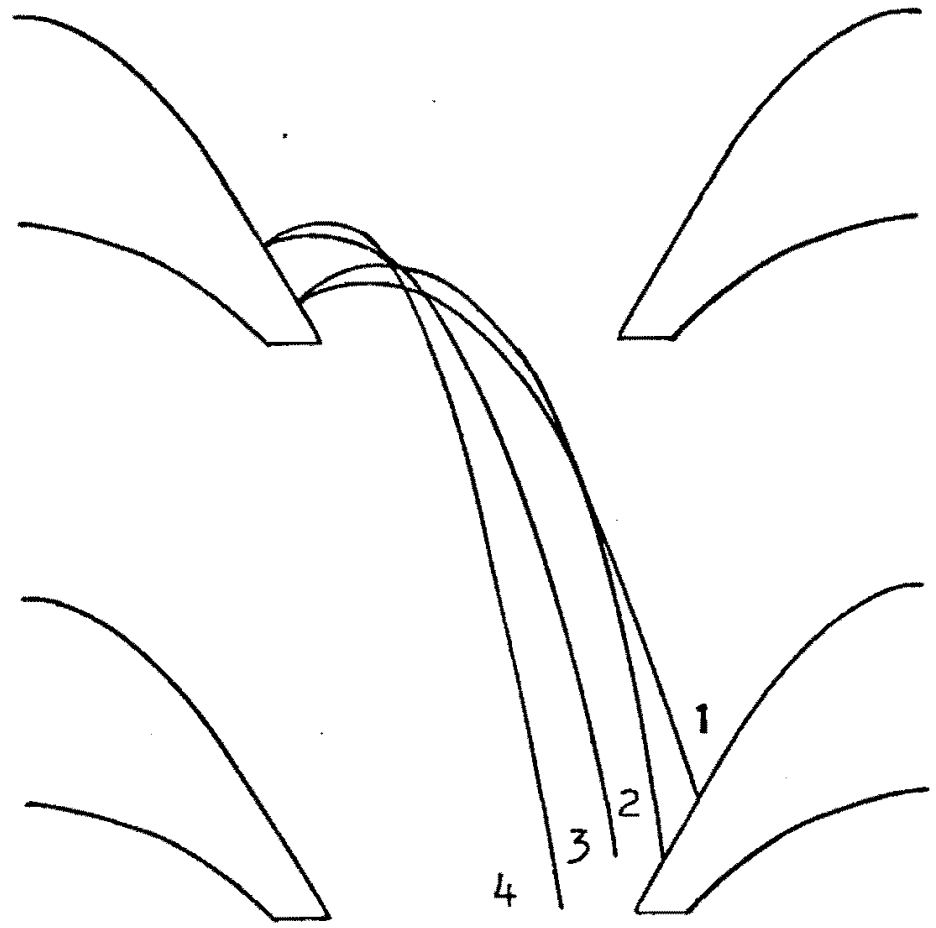

\begin{tabular}{c|cccc}
\hline & $r_{0}$ & $\mathrm{E}$ & $\alpha$ & $\beta$ \\
\hline 1 & 7 & $3 \%$ & $110^{\circ}$ & $180^{\circ}$ \\
2 & 7 & $3 \%$ & $130^{\circ}$ & $180^{\circ}$ \\
3 & 8 & $3 \%$ & $110^{\circ}$ & $180^{\circ}$ \\
4 & 8 & $3 \%$ & $130^{\circ}$ & $180^{\circ}$ \\
\hline
\end{tabular}

Figure 16. Meridional trajectory plots for the 10-unit spacing model for different initial positions and directions. 


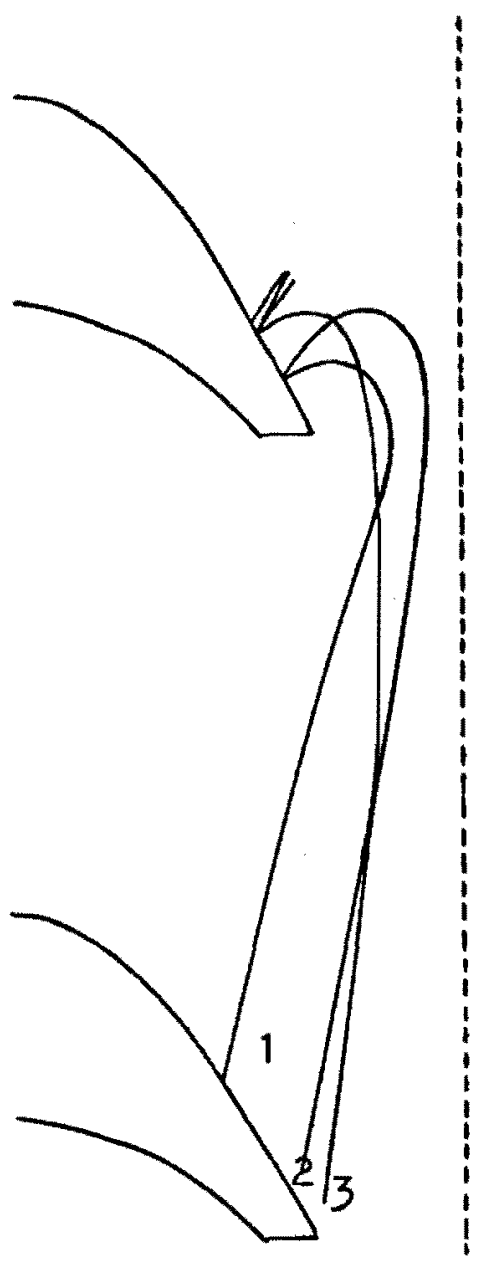

\begin{tabular}{c|cccc}
\hline & $r_{0}$ & $\mathrm{E}$ & $\alpha$ & $\beta$ \\
\hline 1 & 7 & $3 \%$ & $120^{\circ}$ & $210^{\circ}$ \\
2 & 7 & $3 \%$ & $150^{\circ}$ & $200^{\circ}$ \\
3 & 8 & $3 \%$ & $120^{\circ}$ & $210^{\circ}$ \\
4 & 8 & $3 \%$ & $150^{\circ}$ & $190^{\circ}$ \\
5 & 8 & $3 \%$ & $150^{\circ}$ & $200^{\circ}$ \\
\hline
\end{tabular}

Figure 17. Skew trajectory plots for the 10-unit spacing model for different initial positions and directions. 


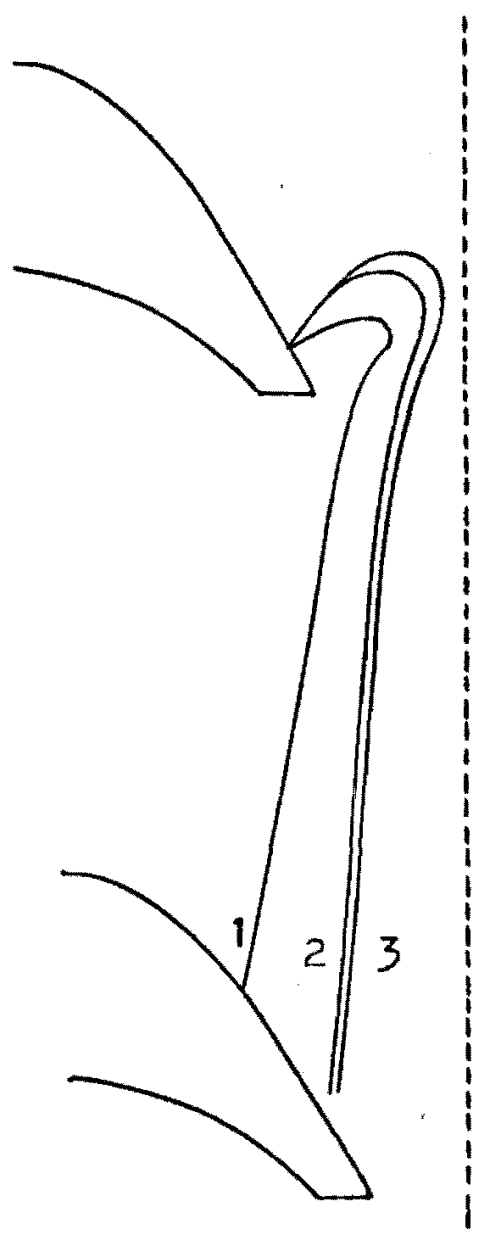

\begin{tabular}{c|cccc}
\hline & $r_{0}$ & $E$ & $\alpha$ & $\beta$ \\
\hline 1 & 7 & $3 \%$ & $120^{\circ}$ & $150^{\circ}$ \\
2 & 7 & $3 \%$ & $150^{\circ}$ & $200^{\circ}$ \\
3 & 7 & $3 \%$ & $150^{\circ}$ & $190^{\circ}$ \\
\hline
\end{tabular}

Figure 18. Skew trajeciory nlots for the 19-unit spacing modei for different initial directions. 
39

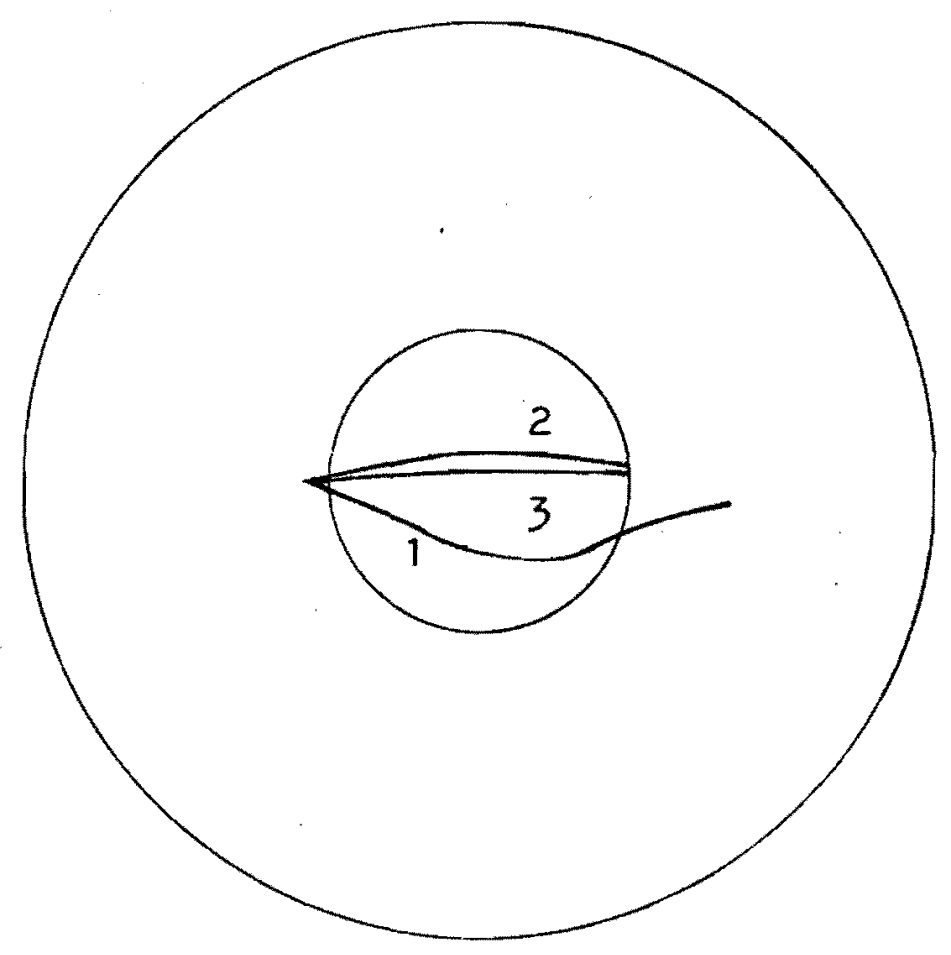

\begin{tabular}{c|cccc}
\hline & $r_{0}$ & $E$ & $\alpha$ & $\beta$ \\
\hline 1 & 7 & $3 \%$ & $120^{\circ}$ & $150^{\circ}$ \\
2 & 7 & $3 \%$ & $150^{\circ}$ & $200^{\circ}$ \\
3 & 7 & $3 \%$ & $150^{\circ}$ & $190^{\circ}$ \\
\hline
\end{tabular}

Figure 19. Skew trajectories projected onto the XY-plane. 


\section{CONCLUSION}

The electron gain of a conical hole type nultiplier structure was studied by computing the potential distribution and some electron trajectories. The only parameter, the influence of which was studied, was the spacing between two successive stages. It was found that the smallest spacing studied of approxinately $77 \%$ of the electrode thickness has two important advantages compared to larger spacings: firstly, the active region where the field strength on the surface pulls the electrons into the vacuum instead of pushing them back to the electrode is wider than in the case of larger spacing; secondly, the pulling field is strong enough to collect electrons with initial energies of more than $5 \%$ of the accelerating voltage, while for larger spacings a smaller fraction of the energy distribution is collected onto the next stage. It is concluded that the conical hole type multiplier structure is feasible with sufficient gain if the spacing is chosen sufficiently small. 


\section{BIBLIOGRAPHY}

Abramowitz, Milton and Irene A. Stegun. Handbook of riathematics Functions. (National Bureau of Standards, Applied Natheratics Series 55.) Washington D.C.: Government Printing Office, 1964. Formula io. 25.2.2, page 878 .

Becker, Richard and Fritz Sauter. Electromagnetic Field and Interactions. Volume 1. New York: Blaisdell Publishing Co., 1964. Formula No. 13.42 , page 39 .

Lenz, Friedrich A. Lectures on Electron Optics. Beaverton, Oregon: Tektronix, Inc., 1968.

Lenz, Friedrich A. A Propram in Fortran IV CCS (Tymshare) Languafe for Electron Trajectories in Axisymetric Electrostatic Fields. Beaverton, Oregon: Tektronix, Inc., 1970.

Southwell, Richard Vynne. Relaxation Methods in Theoretical Physics. (Oxford Engineering Science Series.) Oxford, England: Clarendon Press, 1956. 


\section{APPENDIX A}

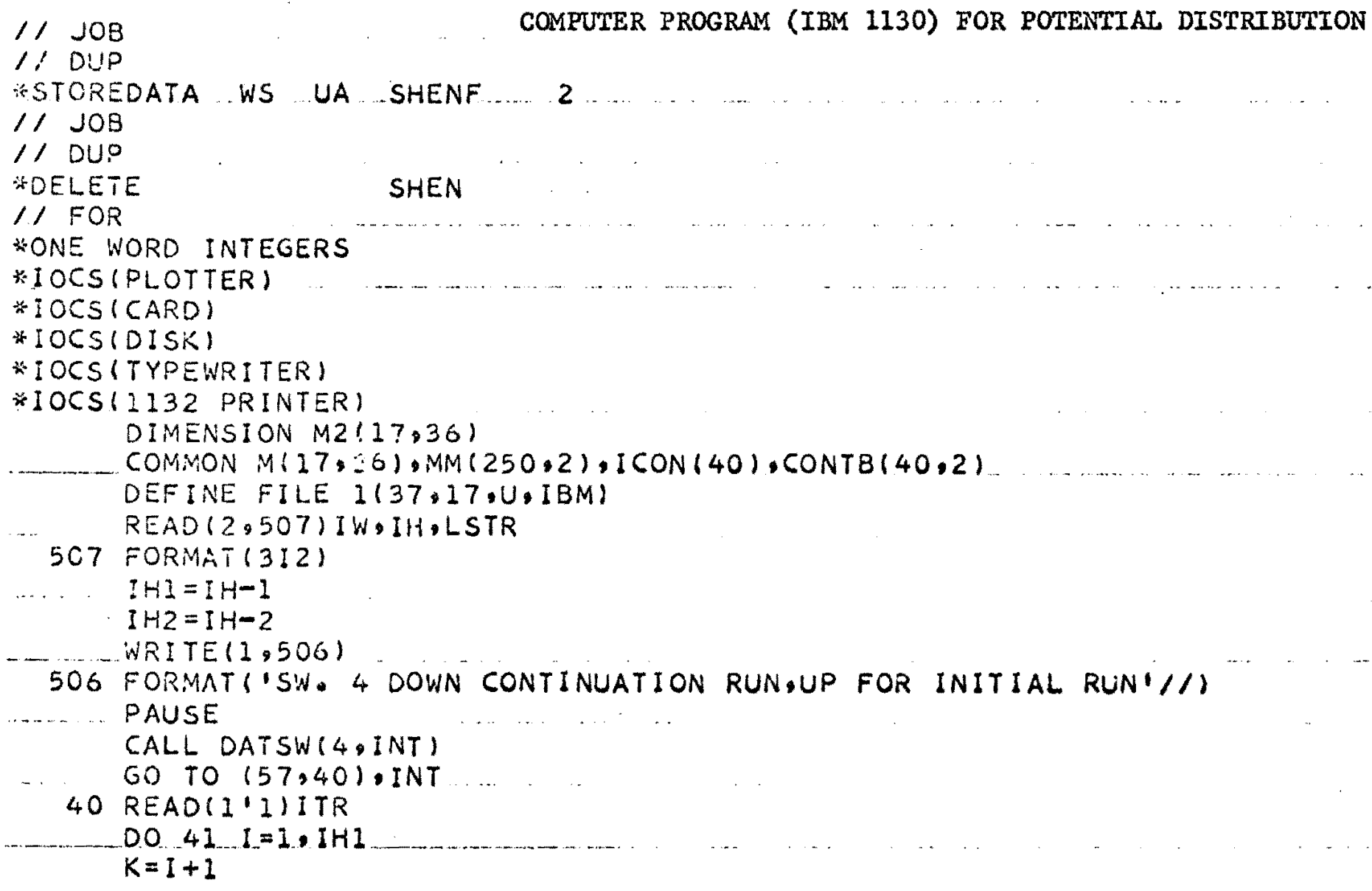

COMPUTER PROGRAM (IBM 1130) FOR POTENTIAL DISTRIBUTION

SHEN 


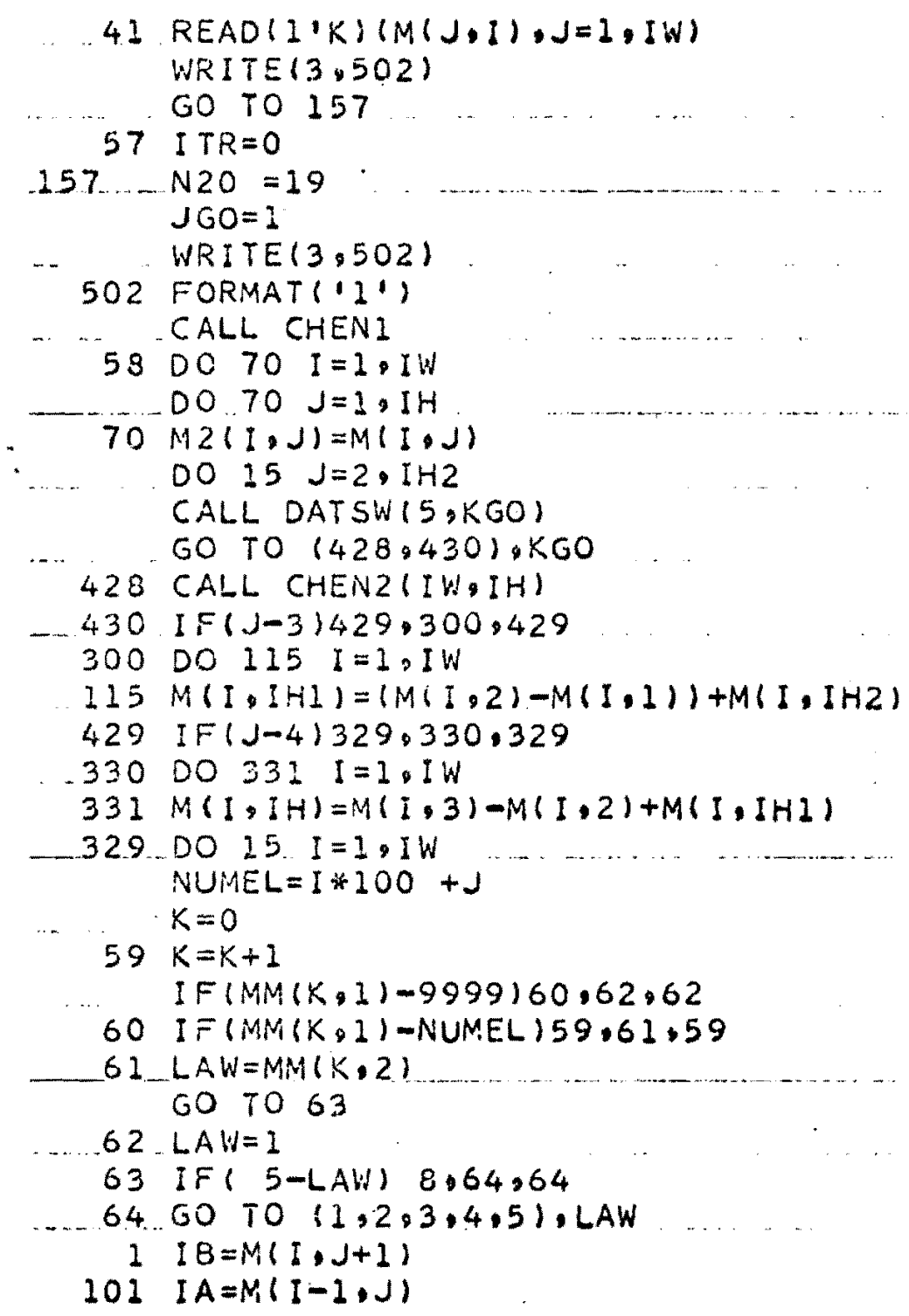


$C=M(? \div 1, J)$

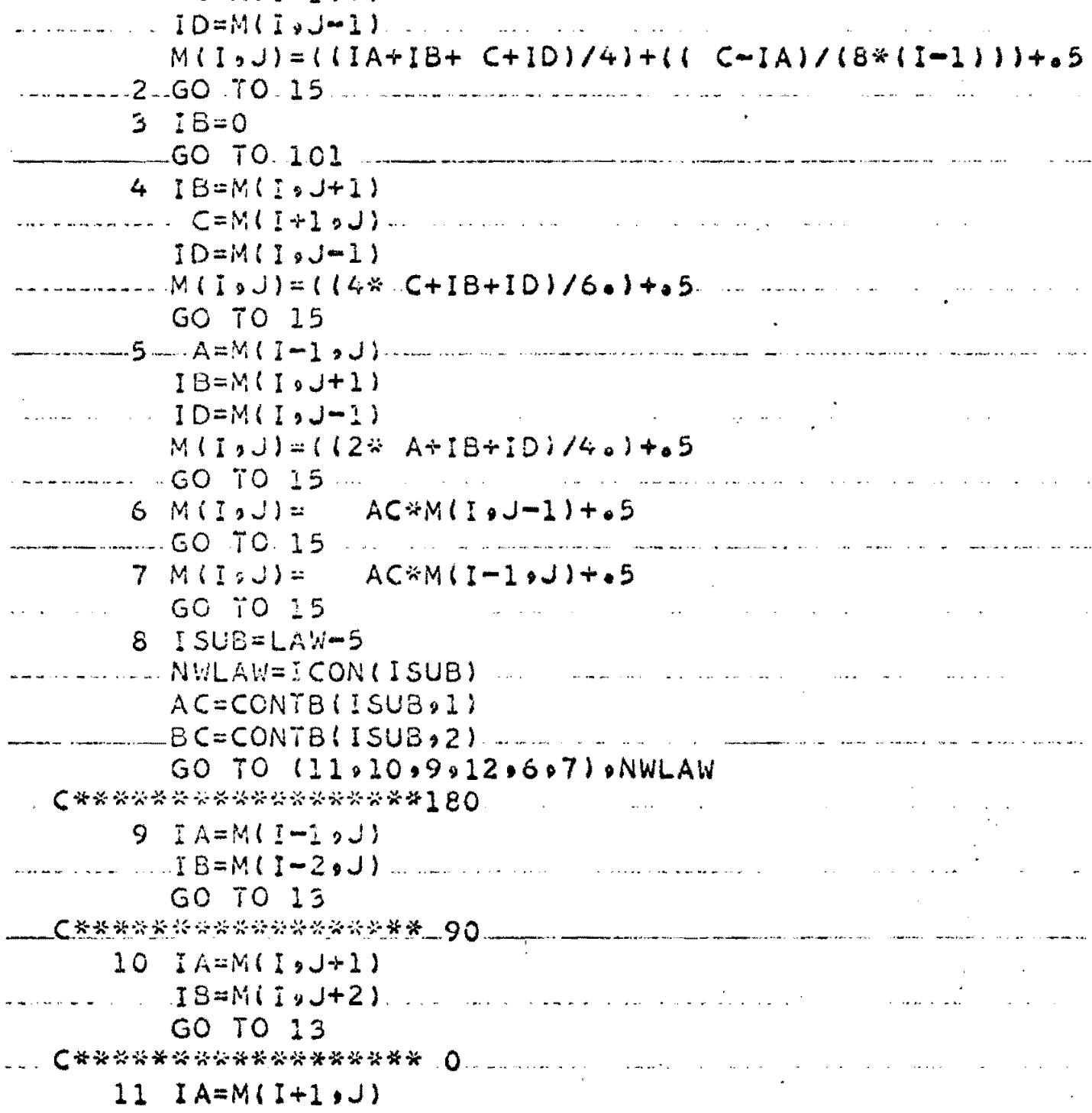

$9 I A=M(I-I, J)$ 


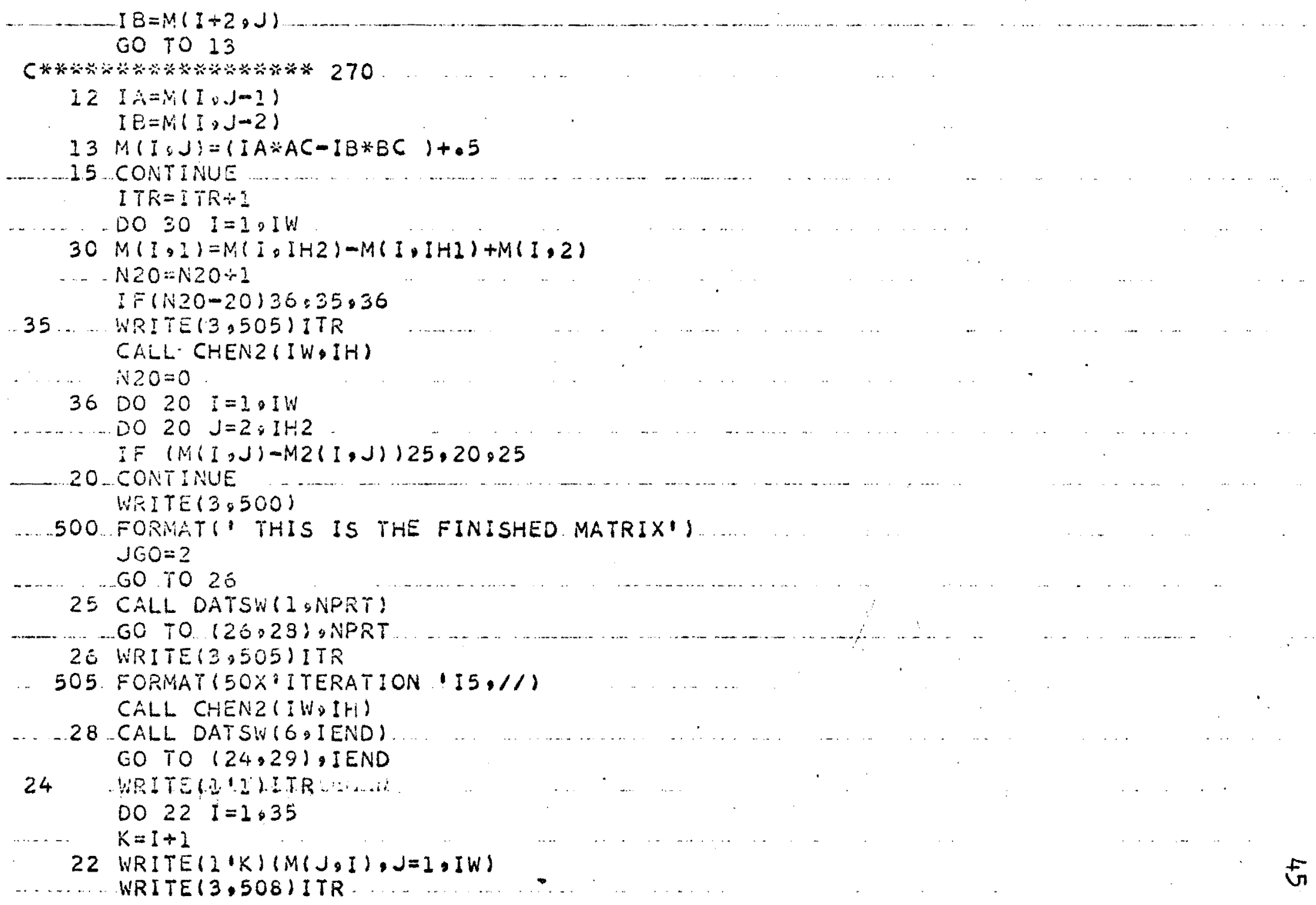


509 FORMAT (20X,?5' IS THE ITR. STORED FOR CONTINUATION RUN $/ 1)$

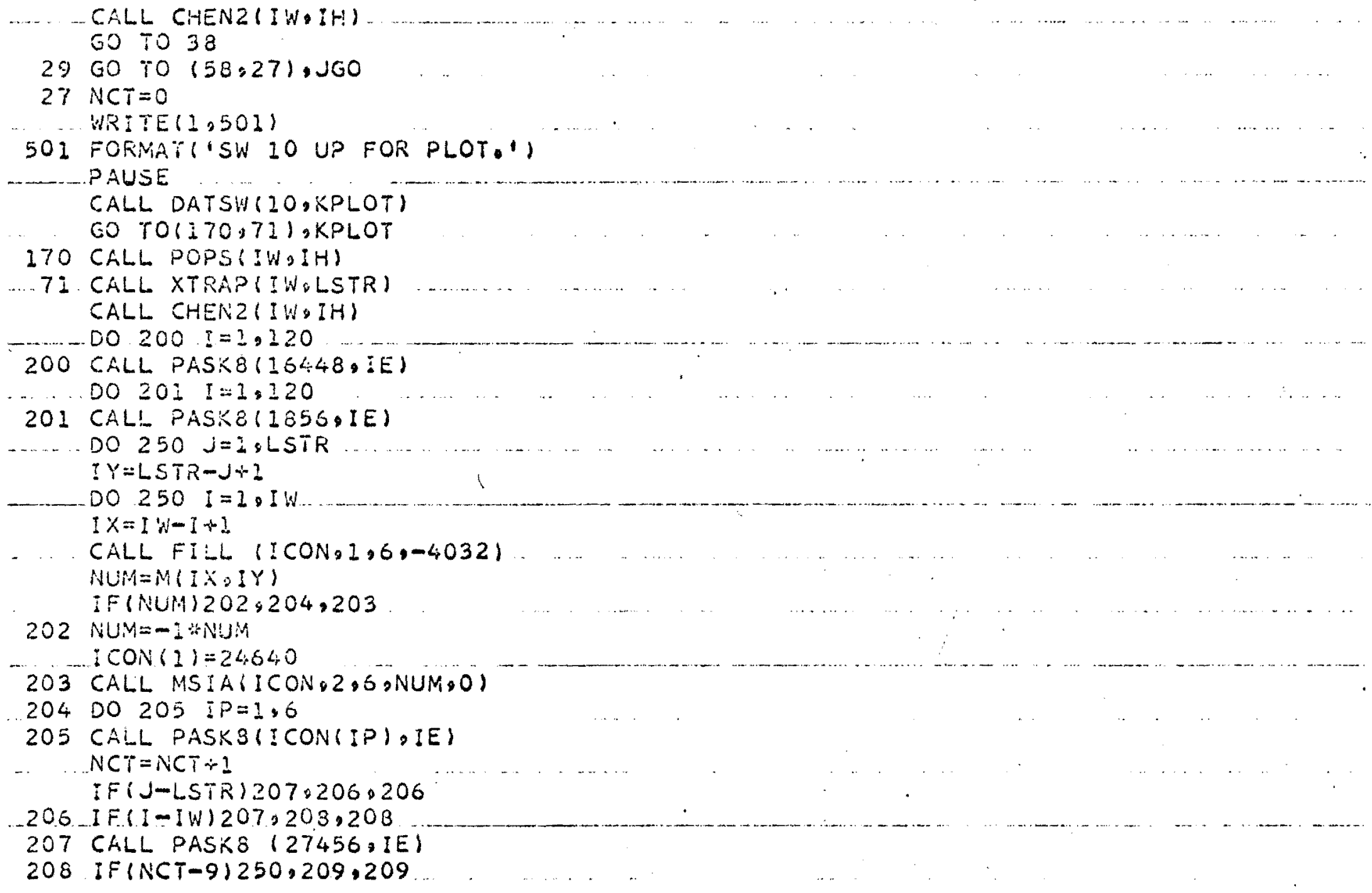




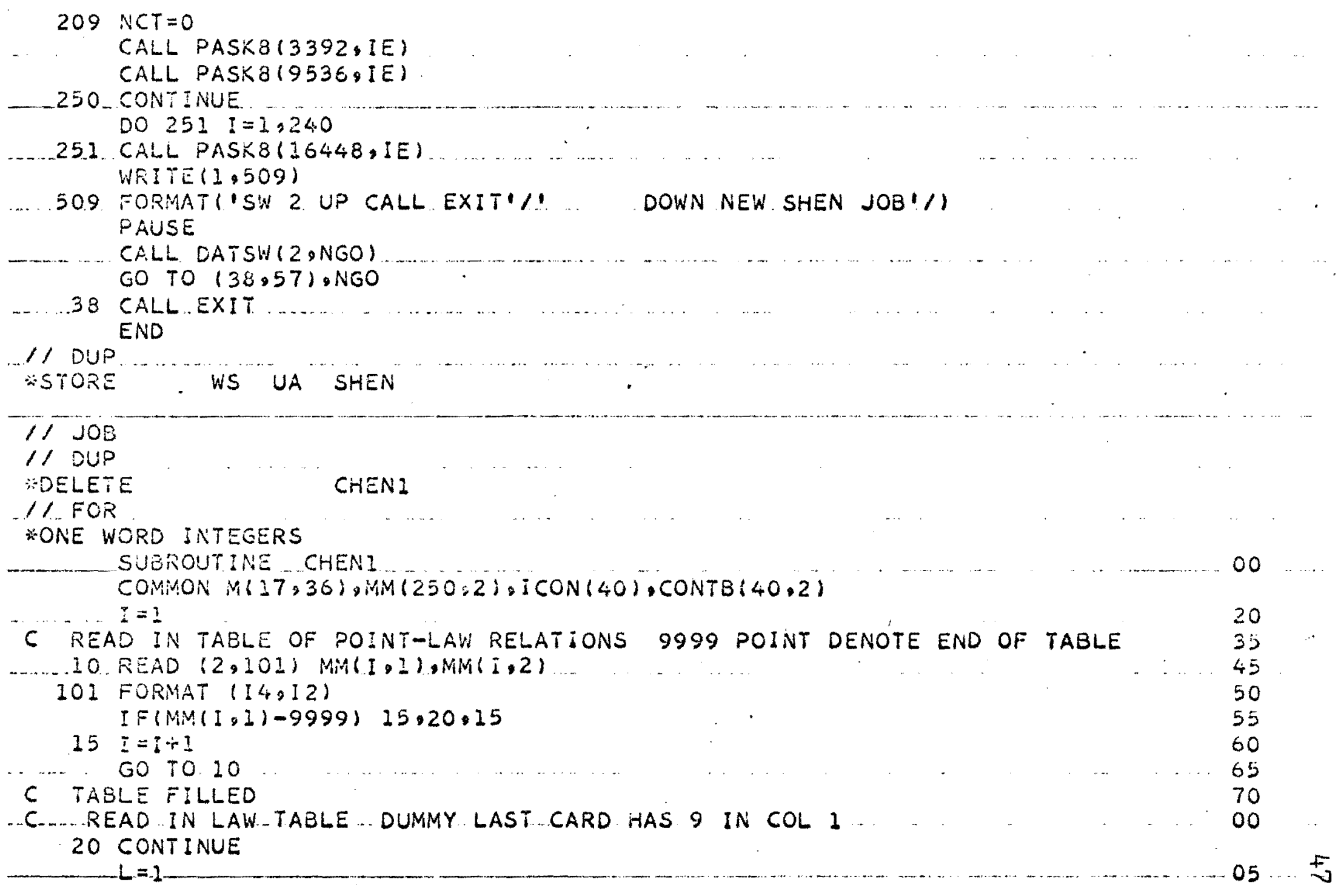




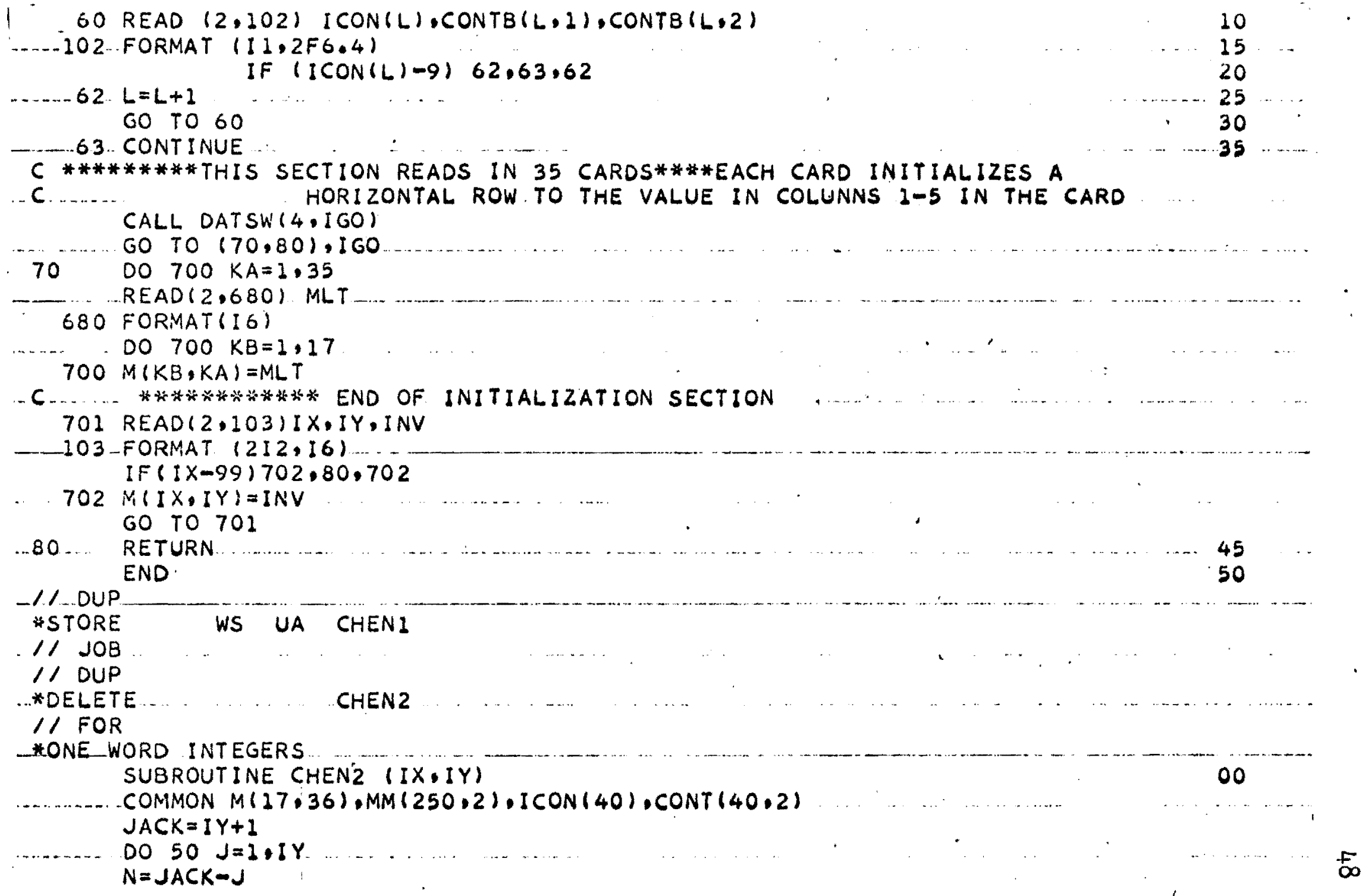




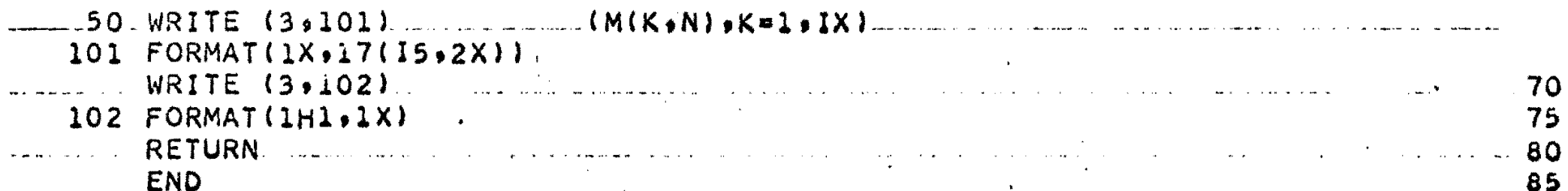

. 11 DUP END

* STORE WS UA CHEN2 
APPENDIX B

POTENTIAL DISTRIBUTION ( IOE-HNIT SPACING)

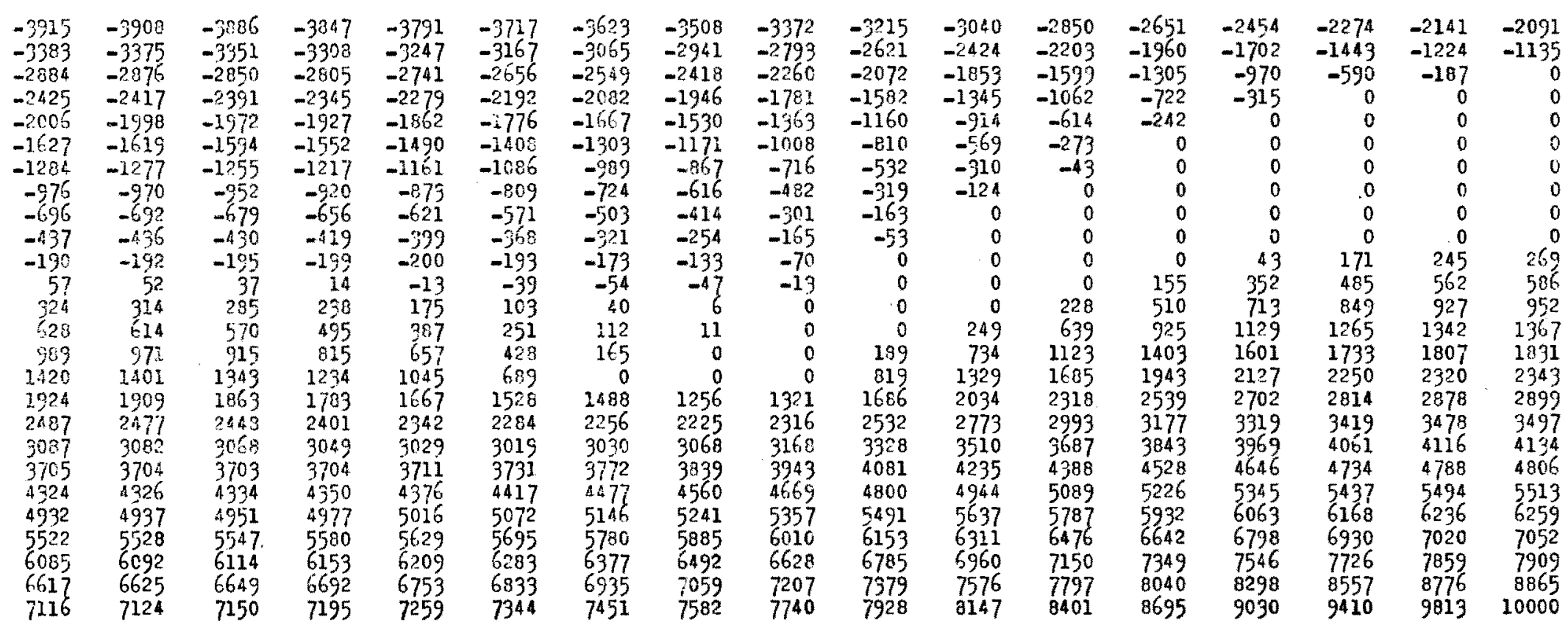


APPENOIX C

POTENTIAL DISTRIBUTION (19-UNIT SPACING)

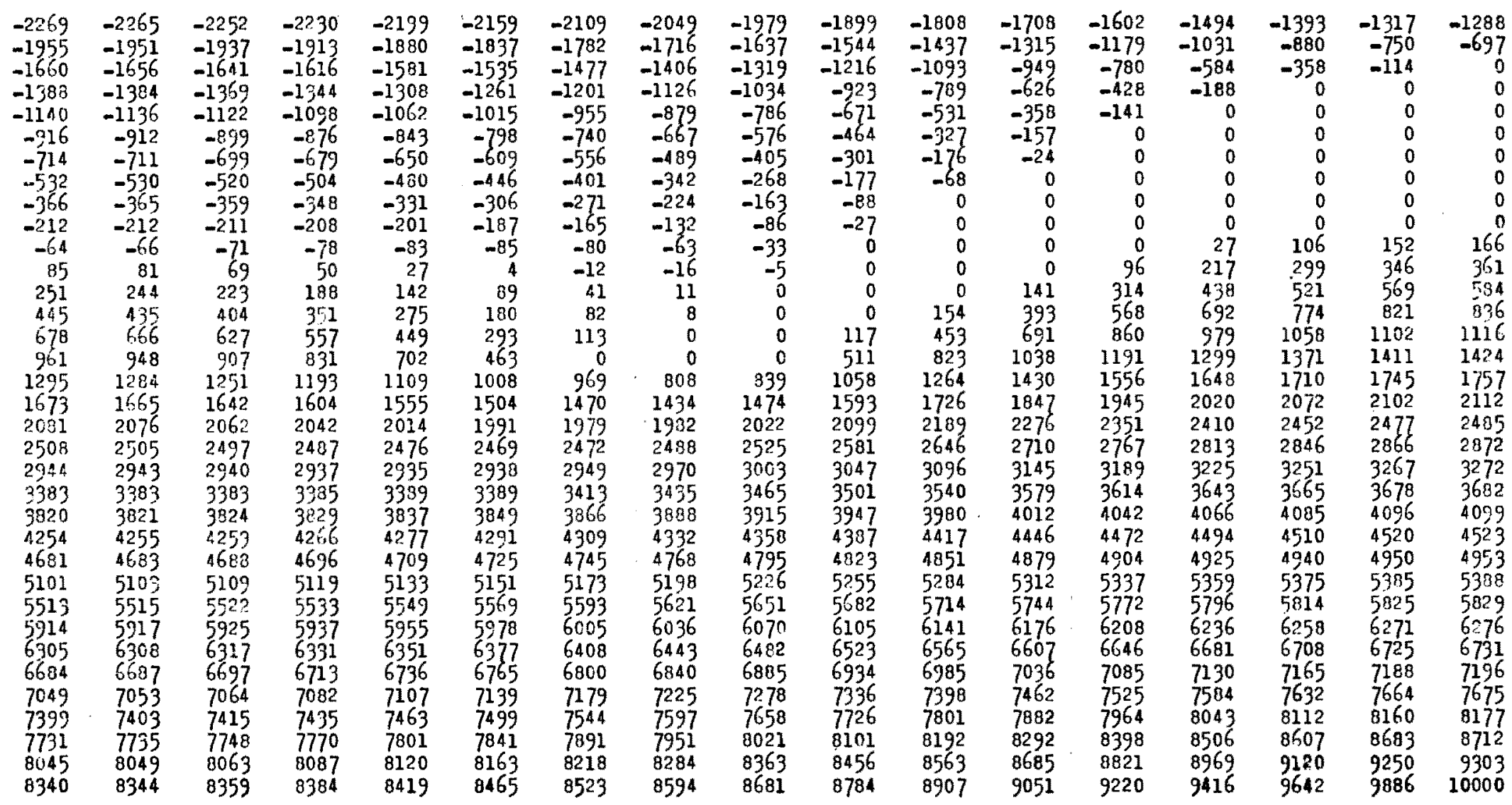


APPENDIX D. POTENTIAL DISTRIBUTION (33-UNIT SPACING)

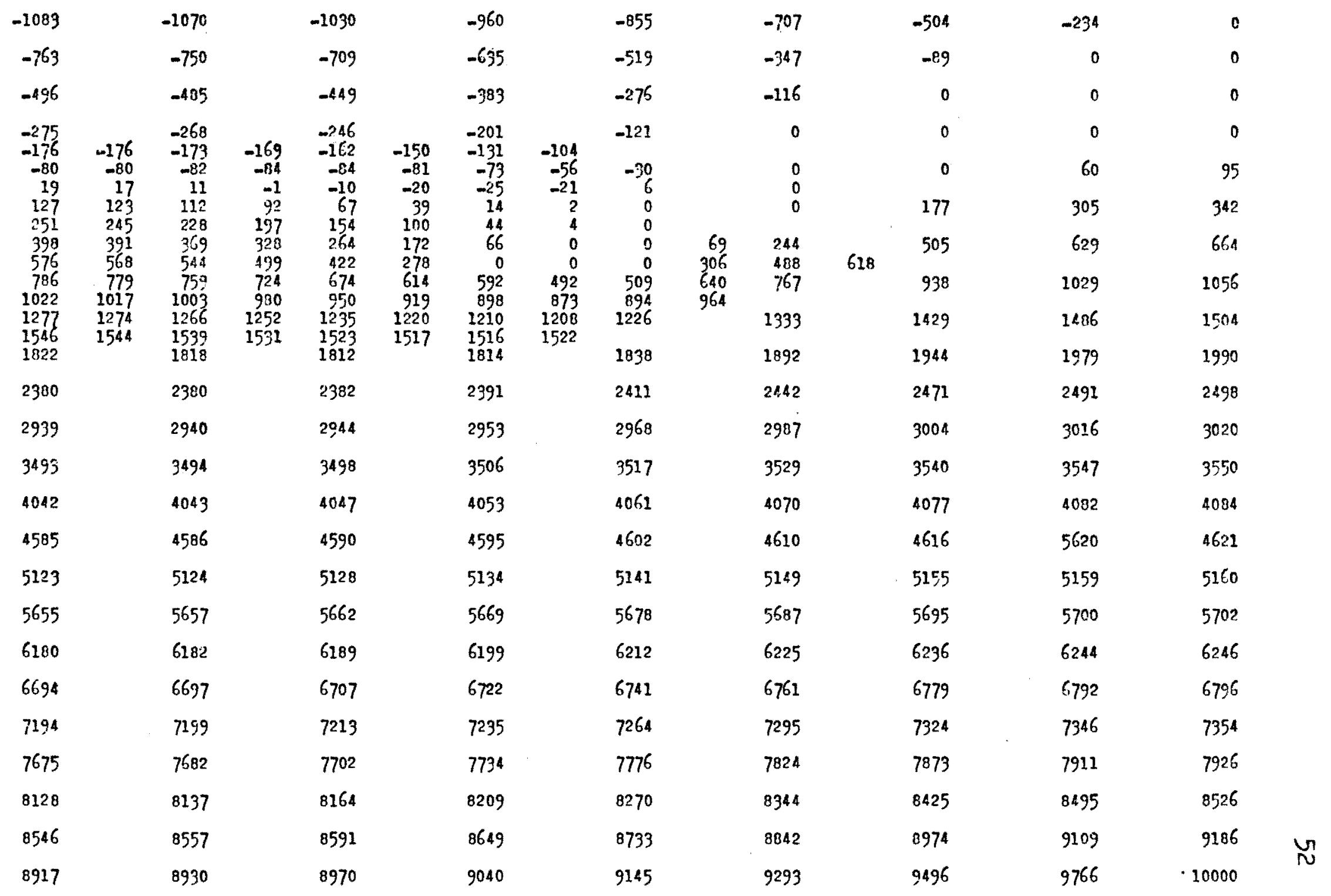

